Please quote as: Kunz, M. M.; Bretschneider, U. \& Leimeister, J. M. (2020): Rewardbased Crowdfunding. In: Kollmann, T. (Ed.), Handbuch Digitale Wirtschaft (1st ed., pp. 755-787). Wiesbaden, Germany: Springer Gabler. 


\title{
Reward-based Crowdfunding
}

\author{
Michael Marcin Kunz, Ulrich Bretschneider und \\ Jan Marco Leimeister
}

\section{Inhalt}

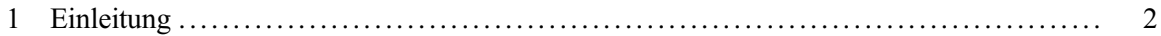

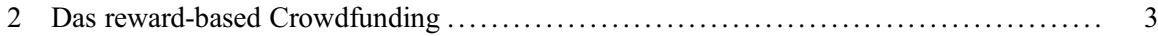

3 Klassifizierung von Erfolgsfaktoren von reward-based Crowdfunding-Projekten ......... 4

4 Methodisches Vorgehen und Expertenbefragung in Anlehnung an die

Delphi-Methode ....................................................... 9

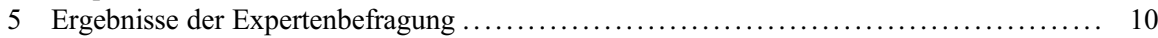

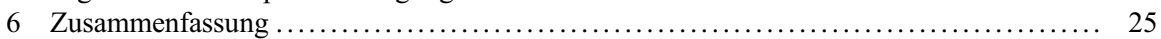

Anhang A.1 Rangfolge der projektspezifischen Erfolgsfaktoren ....................... 29

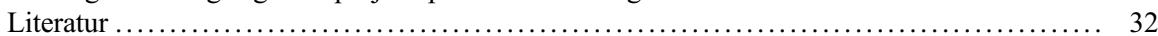

\section{Zusammenfassung}

Crowdfunding unterstützt neue Start-ups dabei, den Finanzierungsbedarf der ersten Unternehmensphase zu überwinden. Dieser Bedarf wird über die Unterstützung einer Vielzahl an Geldgebern mit kleinen Geldbeträgen realisiert. Das Ziel dieses Beitrages ist, den aktuellen Stand der Erfolgsfaktorenforschung im reward-based Crowdfunding zu reflektieren und auf Basis einer qualitativen Expertenbefragung um noch nicht betrachtete Aspekte zu erweitern. Durch dieses Vorgehen erhalten Projektinitiatoren einen Katalog an die Hand, der ihnen dabei hilft die Erfolgschancen eigener Kampagnen zu steigern.

M. M. Kunz $(\bowtie)$

Wirtschaftsinformatik, Universität Kassel, Kassel, Deutschland

E-Mail: mail@michaelkunz.net

U. Bretschneider

Lehrstuhl für Betriebswirtschaftslehre, insbes. Informationsmanagement, FernUniversität in Hagen, Hagen, Deutschland

E-Mail: ulrich.bretschneider@fernuni-hagen.de

J. M. Leimeister

Institut für Wirtschaftsinformatik, Universität St. Gallen, St. Gallen, Schweiz

E-Mail: janmarco.leimeister@unisg.ch 


\section{Schlüsselwörter}

Crowdfunding $\cdot$ Erfolgsfaktoren $\cdot$ Reward-based Crowdfunding $\cdot$ Finanzierung · E-Commerce

Im Zuge jeder Unternehmensgründung ist die Beantwortung einer zentralen Frage unumgänglich: Wie wird das Vorhaben finanziert? Selbst ohne das Vorliegen eines bereits marktreifen Produktes besteht ein grundlegender Kapitalbedarf, verursacht durch etwaige Vorbereitungsmaßnahmen für einen späteren Markteintritt (Kollmann 2005). Die Herausforderung für Entrepreneure liegt im Wesentlichen darin, die Liquidität zu jedem Zeitpunkt sicherzustellen und darüberhinaus die Finanzierungsstruktur entsprechend des Entwicklungsstadiums der Unternehmung mit Blick auf das Wachstum bzw. die Geschäftsentwicklung im Allgemeinen zu optimieren (Fueglistaller et al. 2012). Mit Crowdfunding (CF) als Alternative wurde ein Instrumentarium zur Überbrückung eines Finanzierungsbedarfs der ersten Unternehmensphase geschaffen. CF wird bezeichnet als: „, . . eine Finanzierungsform, die über einen öffentlichen Aufruf initiiert wird (mit oder ohne Intermediär) und zum Ziel hat, finanzielle Mittel für ein Projekt oder Unternehmen mit oder ohne Gegenleistung zu erhalten. Beim Crowdfunding beteiligt sich in der Regel eine größere Zahl von individuellen Kapitalgebern “ (Moritz und Block 2014, S. 60). Solche Gegenleistungen (Belohnung) entsprechen zumeist dem Unterstützungsbeitrag und variieren über Produkte, Dankeschön oder ähnlichen Goodies (Belleflamme et al. 2013a).

Verschiedenste Faktoren können dabei den Erfolg oder Misserfolg einer CFKampagne beeinflussen: Sei es die wahrgenommene Höhe der Projektqualität oder auch die Reichweite der Projektinitiatoren in sozialen Netzwerken (Mollick 2014). Die Palette der Erfolgs- und Misserfolgsfaktoren ist breit gefächert. Die wissenschaftliche Literatur bietet bis zum jetzigen Zeitpunkt hauptsächlich quantitativ erforschte Ergebnisse zu den Erfolgs- und Misserfolgsfaktoren von CF-Projekten. Es besteht daher der Bedarf an qualitativen Forschungsansätzen, die dabei helfen, bestehende Erfolgsfaktoren zu ermitteln, diese zu bewerten sowie in einzelne Themenfelder konkrete Erfolgsfaktoren auszuarbeiten, die letztlich als Grundlage für Handlungsempfehlungen zur erfolgreichen Veröffentlichung von reward-based CF-Kampagnen eingesetzt werden können. Eine solche Bewertung ist zudem von Interesse, da sich das CF kontinuierlich wandelt und sich damit auch die Sicht auf mögliche Erfolgsfaktoren ändern kann, vor allem aufgrund des zunehmenden Erfahrungsschatzes von Unterstützern aus vorangegangen Projekten.

Das Ziel dieses Beitrages ist daher die Konsolidierung bestehender Erfolgsfaktoren des $\mathrm{CF}$, die Ermittlung weiterer Faktoren aus themennahen Feldern sowie die Bewertung dieser auf Basis einer Expertenumfrage unter Einbezug der vier zentralen Interessengruppen im CF: Unterstützer, Projektinitiatoren, $\mathrm{CF}-$ Wissenschaftler und CF-Berater. Der Fokus der Untersuchung liegt auf dem reward-based CF, da diese Art des CF aus unterschiedlichster Hinsicht - wie Nutzerzahlen, Projektzahlen, Umsatzzahlen - von Bedeutung ist. 


\section{Das reward-based Crowdfunding}

Beim reward-based CF können sich Investoren an Kampagnen und Produkten beteiligen und erhalten anstelle einer finanziellen Gegenleistung oftmals Belohnungen (Rewards) für ihre Beteiligung. Solche Belohnungen sind von der Höhe der finanziellen Beteiligung abhängig. Größere Beiträge werden vielmals mit limitierten und exklusiven Rewards belohnt (Dragonetti und Weiss 2015). So kann es sich bei den entsprechenden Belohnungen beispielsweise um eine ideelle Widmung oder auch um konkrete Produkte handeln, welche durch eine Vorfinanzierung der Kampagne produziert werden. Beim Modell des reward-based CF spricht man auch vom Pre-sales Modell, wobei von den Unterstützern finanzielle Mittel für die Herstellung eines Produkts bereits vor dem Verkauf bereitgestellt werden (Blohm et al. 2015). Ist die Unterstützung mit einer Vorbestellung des Produkts als Gegenleistung gekoppelt, wird der Verkaufspreis des Produkts im Vergleich zu dem im herkömmlichen Handel erniedrigt (Sixt 2014).

Bei der hier betrachteten CF-Spielart sind die Finanzierungsmechanismen klar definiert (Blohm et al. 2015). Der All-or-Nothing Finanzierungsmechanismus dominiert bei reward-based Plattformen. Dies bedeutet, dass eingesammelte Beträge aus Kampagnen erst nach erfolgreichem Erreichen der Finanzierungssumme und zugleich nach Ablauf der Kampagnenlaufzeit an die Projektmitglieder ausgezahlt werden. Wird die Finanzierungssumme nicht erreicht, so werden die Beträge an die Unterstützer zurückgezahlt. Im Gegensatz hierzu steht der Finanzierungsmechanismus Keep-it-All. Hiernach muss keine bestimmte Finanzierungssumme erreicht werden, um den entsprechenden Betrag ausgezahlt zu bekommen.

Der Ablauf einer Kampagne im reward-based CF ist gekennzeichnet durch drei unterschiedliche Prozessschritte. In Abhängigkeit von dem jeweiligen Projektfinanzierungsstatus untergliedert sich dieser CF-Prozess in die Phasen vor, live und nach der Finanzierung. In der Kampagnen-Vorphase erfolgt die gesamte und detaillierte Planung zur Kampagne sowie zur Projektumsetzung. Dabei gilt es so detailliert wie möglich die Planung vorzunehmen, um einen Erfolg der Kampagne sicherstellen zu können. Die Live-Phase definiert den Zeitraum zwischen dem Go-Live und dem Kampagnenende nach Erreichen der Laufzeit. Innerhalb dieser Phase werden durch die Initiatoren Maßnahmen ergriffen, die eine Sicherstellung der Zielerreichung Erreichen des Finanzierungsbetrages - ermöglichen. In der nachgelagerten Phase, nach Kampagnenende, wird bei nicht erfolgreichem Abschluss die Kampagne rückabgewickelt. Bei erfolgreichem Abschluss hingegen gehen die Initiatoren in die Umsetzungsphase und realisieren das beworbene Produkt bzw. die beworbene Dienstleistung.

Zielgruppe von Kampagnen im reward-based CF sind hauptsächlich private Investoren. Institutionelle Investoren sind kaum vertreten. Bei den Projektgründern handelt es sich sowohl um Privatpersonen als auch Institutionen. Es ist zudem festzuhalten, dass es sich beim reward-based CF, trotz der Nähe zum E-Commerce in Form eines Marktplatzes, um ein risikobehaftetes Geschäft handelt. Die getätigten Finanzierungen führen nicht zwangsläufig zum letztendlichen Erfolg einer Kampagne. Auch nach erfolgreicher Kampagnendurchführung können in der Realisie- 
rungsphase Probleme auftreten, die zum nachträglichen Scheitern einer Kampagne führen können, womit die getätigten Finanzierungsbeiträge der Unterstützer in den meisten Fällen als Verlust zu deklarieren sind.

\section{$3 \quad$ Klassifizierung von Erfolgsfaktoren von reward-based Crowdfunding-Projekten}

Die Kampagne ist objektiv betrachtet dann erfolgreich, wenn das angestrebte Finanzierungsziel erreicht wurde. Innerhalb dieser Studie wird genau diese objektive Betrachtung als Anhaltspunkt für den Erfolg einer Kampagne angenommen. Der Erfolg von CF-Kampagnen kann in einige wesentliche Parameter gegliedert werden.

\subsection{Klassifizierung von Erfolgsfaktoren}

Zur Ermittlung der Erfolgsfaktoren zum reward-based CF wurde eine umfassende Literaturrecherche in Anlehnung an Webster und Watson (2002) vorgenommen. Dazu wurden neben der Recherche im Themengebiet des CF auch themennahe Forschungsgebiete aufgenommen. Dazu gehören $u$. a. Theorien rund um das Kaufverhalten und die Online-Produktpräsentation im Rahmen des E-Commerce. Zudem wurden in die Ermittlung der Erfolgsfaktoren auch die jeweiligen Richtlinien und FAQ's der reward-based CF-Plattformen Startnext, Kickstarter und Indiegogo herangezogen.

Im Folgenden werden die drei ermittelten Erfolgsfaktoren klassifiziert und näher beschrieben: personenspezifische Erfolgsfaktoren, Erfolgsfaktoren der Projektgestaltung und projektspezifische Erfolgsfaktoren.

\subsubsection{Personenspezifische Erfolgsfaktoren}

Personenspezifische Erfolgsfaktoren hängen direkt oder indirekt mit dem Projektinitiator selbst bzw. den einzelnen Mitgliedern des Projektteams zusammen.

Ein soziales Netzwerk eines Projektinitiators, insbesondere die Größe des sozialen Netzwerks, das über Facebook oder Twitter erreicht werden kann, spielt eine zentrale Rolle (Mollick 2014). Begründet wird dies damit, dass Kontakte aus dem persönlichen Netzwerk ein stärkeres Interesse am Erfolg der CF-Kampagne haben und somit die Wahrscheinlichkeit einer Finanzierung steigt.

Ein weiterer Erfolgsfaktor ist der Bildungsgrad bzw. die Qualifikation eines Unternehmers als Signal für unternehmerische Produktivität oder Innovationen (BackesGellner und Werner 2007). Die Untersuchung von Ahlers et al. (2015) zeigt, dass im Kontext des equity-based CF der Bildungsstand der Initiatoren eines Start-ups positive Auswirkungen auf die Investition und die Anzahl der Investoren hat. Als Indikator für einen hohen Bildungsstand diente in der Untersuchung ein MBA-Abschluss, da der MBA-Abschluss im Allgemeinen eine gewisse Qualifikation, Berufserfahrung und Reife voraussetzt. 
Zheng et al. (2014) bestätigen in ihrer Arbeit die Hypothesen, dass die Erfahrungen des Teams positiv mit der pünktlichen Auslieferung der Gegenleistung und der Produktqualität verbunden sind. Die Produktqualität beschreibt hier das Ausmaß, in dem die Produktspezifikationen dem ausgelieferten Produkt/Service entsprechen. Im Rahmen des reward-based CF spiegelt das ausgelieferte Produkt die Gegenleistung wider.

Die Autoren Zvilichovsky et al. (2013) betrachteten in ihren Untersuchungen die Wirkung der Unterstützung fremder Projekte auf den eigenen Projekterfolg. Nach ihren Ausführungen stieg mit jedem unterstützten Projekt die Erfolgswahrscheinlichkeit der eigenen CF-Kampagne. Zudem zeigte sich auch, dass die Anzahl der Unterstützer als auch das Fundingziel mit steigender Unterstützungsaktivität des Initiators steigen. Zu ähnlichen Ergebnisse gelangen Younkin und Kashkooli (2013). Für potenzielle Unterstützer stellen vergangene Erfolge ein Signal für die Qualität des Initiators dar.

Zum erfolgreichen Kampagnenabschluss ist es zentral, dass der Initiator aktiv mit der Crowd interagiert. So bieten die meisten Plattformen verschiedene Möglichkeiten wie Chats, Blogs und Kommentarfunktionen an, um aktiv mit der Crowd zu kommunizieren. Unterschiedliche Quellen zeigen auf, dass der Erfolg einer Kampagne maßgeblich von der Kommunikationsaktivität des Initiators abhängt. Dabei umfasst die Kommunikationsaktivität nicht nur die Kommunikation mit der Crowd, sondern auch intensive Presse- und Öffentlichkeitsarbeit.

\subsubsection{Erfolgsfaktoren der Projektgestaltung}

Die Erfolgsfaktoren der Projektgestaltung beziehen sich auf die visuelle und inhaltliche Gestaltung der CF-Kampagne.

Eine Projektbeschreibung sollte ansprechend und kreativ gestaltet sein, um so Begeisterung für das Projekt zu erwecken. Eine klare Formulierung der Inhalte, transparente Darstellung des Verwendungszwecks des Kampagnenbetrages und eine persönliche sowie authentische Kommunikation helfen dabei, die Kampagne erfolgreich zu machen. Laut Mollick (2014) sollte eine Projektbeschreibung zudem frei von Rechtschreibfehlern sein, da diese zu einer reduzierten Wahrnehmung von Einsatzsatzbereitschaft und Qualität führen können.

Auf Basis der E-Commerce Literatur zur Produktpräsentation konnte die Visualisierung der Kampagne als ein weiterer Kernpunkt der Projektgestaltung festgemacht werden (Lim et al. 2013). Die Visualisierung bezieht sich auf die Präsentation der Gegenleistungen mit Texten, Bildern und Videos. Dabei spielen die Interaktivität, Lebendigkeit und soziale Präsenz eine bedeutende Rolle. Eine interaktive Produktpräsentation hilft einem Unterstützer dabei, den Nutzen eines Produkts besser erfassen zu können. Lebendigkeit hingegen ermöglicht den Unterstützern, die Produktinformationen über mehrere Sinnesorgane aufzunehmen. Die soziale Präsenz beschreibt das Ausmaß, in dem der Unterstützer eine Umgebung wahrnimmt, die menschliche Wärme und Geselligkeit vermittelt. Untersuchungen zeigen, dass diese Aspekte positive und signifikante Auswirkungen auf die Intentionen eines Kaufs auf einer Webseite haben.

Die Nutzung von Videos stellt eines der zentralen Informationsmedien innerhalb einer Kampagne dar. Diesen Videos wird eine hohe Bedeutung zugeschrieben. 
Mollick (2014) sieht das Pitch-Video als ein Qualitätssignal, welches die Einsatzbereitschaft des Initiators zeigt. Die Erfolgswahrscheinlichkeit für eine Kampagne mit Video ist signifikant größer als für Kampagnen ohne Video (Gafni et al. 2018). Eine Videodauer von 3-4 Minuten wird empfohlen (Kunz et al. 2017), um die Aufmerksamkeitsspanne nicht zu überschreiten. Auch der Videostory, als eine Art authentische Darstellung der Geschichte und Idee, die hinter der Kampagne steckt, wird eine wichtige Rolle beigemessen (Kuppuswamy und Bayus 2018).

Laut Dual Coding Theory von Paivio (1990) gibt es zwei kognitive Subsysteme. Das eine ist spezialisiert auf die Verarbeitung von verbalen und das andere auf die Verarbeitung von visuellen Informationen. Die zentrale Erkenntnis, die aus der Theorie hervorgeht ist der „picture superiority effect“. Dieser Effekt besagt, dass Bilder sowohl verbal als auch visuell verarbeitet werden, während es für Texte weniger wahrscheinlich ist, dass sie im visuellen System gespeichert werden. Daraus ergibt sich auch die Überlegenheit von Bildern gegenüber Texten, wenn es um das Erinnern an Informationen geht (Hong et al. 2004).

\subsubsection{Projektspezifische Erfolgsfaktoren}

Die projektspezifischen Erfolgsfaktoren beziehen sich auf das Projektvorhaben. Innerhalb der projektspezifischen Erfolgsfaktoren werden grundlegende Entscheidungen (wie z. B. das Fundingziel, die Laufzeit) und Ergebnisse (wie z. B. die Projektidee und der Businessplan) festgehalten, auf denen die Kampagne aufbaut.

Die Projektidee bildet das Herzstück einer Kampagne. Ziel des Initiators ist es, die Crowd von seiner Idee zu überzeugen. Harzer (2013) konnte innerhalb einer Befragung von potenziellen Unterstützern zeigen, dass die Projektidee für die Entscheidung, eine Kampagne zu unterstützen, nur eine geringe Bedeutung hat. Jedoch kann die Projektidee an sich den Erfolg beeinflussen. Innovative Projektideen, die der Crowd einen klaren Mehrwert liefern und sich von anderen Projekten abheben, sind am erfolgversprechendsten. Auf Basis des Innovationsgrades einer Kampagne können Initiatoren sich darüberhinaus von anderen Kampagnen abgrenzen und somit einen Widererkennungswert für ihre eigene Kampagne schaffen (Mollick und Kuppuswamy 2014). Der Schwierigkeitsgrad einer Kampagne, bezogen auf das mögliche Erreichen von Zielen und die Erfüllung der Kampagne, zeigt, dass ein hoher Schwierigkeitsgrad den Projekterfolg negativ beeinflussen kann (Tatikonda und Rosenthal 2000). Projekte mit hohem Schwierigkeitsgrad können sich in hohen Produktstückkosten, unerwünschten Ergebnissen und/oder niedriger Produktqualität bemerkbar machen. Ergebnisse zeigen, dass ein hoher Schwierigkeitsgrad zu einer verspäteten Auslieferung und reduzierten Qualität der Gegenleistungen führt (Zheng et al. 2014).

Innerhalb einer Kampagne spielt zudem der soziale Aspekt eine zentrale Rolle. Dies spiegelt sich in der Zusammenstellung des Teams, der Wirkung von Familie und Freunden, Anzahl der Unterstützer sowie der Community selbst wider (Gafni et al. 2018). Projekte, hinter denen ein Team steht, sammeln mehr Kapital ein, als Projekte mit nur einem Projektinitiator. Dies basiert auf der Tatsache, dass größere Teams unterschiedliche Fähigkeiten zur Verfügung stehen, die eine Aufteilung der Arbeitslast eines solchen Projektes ermöglichen. Ein größeres Team suggeriert mehr 
Glaubwürdigkeit und Vertrauen gegenüber einer einzelnen Person. Die Gruppe der Familie und Freunde sind ein wichtiger Bestandteil innerhalb der ersten Phase einer Kampagne, da sie die Kampagne vom Start an, durch eigene Investitionen sichtbar nach vorne bringen und damit Dritten gegenüber die Kampagne indirekt bewerben können.

Die Anzahl an Unterstützern einer Kampagne pro Tag ist ein wichtiger Faktor einer laufenden Kampagne, vor allem in der Startphase. Prefundia (2013) zeigt hierzu, dass 50 Unterstützer am ersten Tag der Kampagne 50 Unterstützern pro Tag entsprechen, aber 50 Unterstützer am fünften Tag der Kampagne nur noch 10 Unterstützern entsprechen. Daraus zieht der Autor das Fazit, bereits vor der Finanzierungsphase eine Gefolgschaft aufzubauen, damit möglichst viele Unterstützer am ersten Tag der Kampagne ihren Beitrag leisten. Nicht nur die Plattformbetreiber messen der Anzahl der Unterstützer eine hohe Bedeutung bei. So stellt Harzer (2013) in einer quantitativen Befragung von potenziellen Unterstützern fest, dass die Anzahl bisheriger Unterstützer den Erfolg einer CF-Kampagne positiv beeinflusst.

Um im CF eine Kampagne erfolgreich abschließen zu können, ist der Aufbau einer Community, die den Initiator unterstützt, von großer Bedeutung (Belleflamme et al. 2013b). Eine Community aufzubauen erfordert vor allem die Integration der sozialen Online-Netzwerke in die unternehmerischen Prozesse als Mittel, um mit der Crowd zu interagieren. Ist erstmal eine Community aufgebaut, können die Beteiligten wesentliche Vorteile sog. „community benefits“ genießen. Beim reward-based CF profitiert der Initiator von der Crowd, die das Produkt mit ihrem Unterstützungsbeitrag vorbestellt. Der Initiator kann hier eine Preisdiskriminierung zwischen den Crowdfundern und den regulären Verbrauchern machen, die das Produkt erst kaufen können, wenn es am Markt ist. Damit genießen Unterstützer eine Nutzensteigerung, weil sie das Gefühl schätzen, zu einer privilegierten Gruppe zu gehören, die zur Existenz des Produkts beigetragen hat. Zudem wirkt sich die Beteiligung eines Projektinitiators innerhalb der Community positiv auf die Erfolgswahrscheinlichkeit und die Höhe der erzielten Fundingsumme aus (Zvilichovsky et al. 2013). So entstehen im CF neue soziale Interaktionen, die eine Crowd dazu motivieren, Projekte zu unterstützen, die ein Gefühl der Verbundenheit mit einer Community mit ähnlichen Interessen und Idealen bieten (Gerber et al. 2012).

Um während und vor allem nach erfolgreichem Abschluss der Kampagne weiterhin erfolgreich zu sein, müssen Initiatoren vorbereitet sein, im Sinne von Business- und Zeitplänen. Solche Projekte haben eine größere Wahrscheinlichkeit, das Produkt rechtzeitig auszuliefern und zusätzliche Vorteile zu erlangen (Mollick und Kuppuswamy 2014). Ein gewisser Grad an Projektplanung ist nötig, um Projektunsicherheiten zu reduzieren und die Erfolgswahrscheinlichkeit zu erhöhen (Zheng et al. 2014). Die konsequente Verfolgung und Überwachung eines Projekts anhand des Projektplans kann sicherstellen, dass das finale Produkt pünktlich und innerhalb des Budgets ausgeliefert wird.

Eine solche Planung sollte auch von Vorneherein definieren, welche Gegenleistungen angeboten und wie diese auf der Kampagnenseite umgesetzt werden, im Sinne von Pledge-Levels. Ersteres beschreibt dabei die Gegenleistung für eine getätigte finanzielle Unterstützung. Solche Gegenleistungen variieren von formalen 
Dankeschöns und steigen mit dem Unterstützungsbetrag, z. B. von der Möglichkeit exklusiv die ersten Produkte der Kampagne zum Vorzugspreis zu erhalten, einem Treffen mit dem Initiator bis hin zu einer Rolle in einem Film (Mollick 2014). Je kreativer die Gegenleistungen ist, umso erfolgsversprechender wird die Kampagne gesehen. In diesem Zusammenhang wird auch die Bedeutung des Angebotsumfangs, im Sinne unterschiedlicher Pledge-Levels, angesprochen. So sind auf der Plattform Kickstarter Kampagnen, die niedrige Pledge-Levels mit weniger als 20 US-Dollar anbieten, erfolgreicher als die Kampagnen, die keine Pledge-Levels unter 20 USDollar anbieten (Strickler 2010). Deshalb wird empfohlen, eine breite Staffelung der Pledge-Levels anzubieten.

Ein umfassender Kommunikationsplan beinhaltet die Summe geplanter Maßnahmen, um eine Kampagne bekannt zu machen (Nastoll 2013). Das Ziel der Erstellung eines Plans ist das frühzeitige Auseinandersetzen des Initiators mit möglichen Quellen der Kommunikation und Promotion. Als wichtiges Werkzeug zur schnellen und weitreichenden Verteilung von Informationen werden soziale Medien wie Facebook und Twitter gesehen (Xu et al. 2014). Neben der Promotion über die Online-Kanäle, ist es auch von Vorteil andere Kanäle wie TV oder Radio zu nutzen (Lu et al. 2014). Auch wenn die Promotion der Kampagnen hauptsächlich Online stattfindet, müssen demografische Eigenschaften beachtet werden. Denn mit zunehmendem Alter nehmen die Aktivitäten in Online-Communities ab (Harzer 2013).

Um den Informationsfluss stetig aufrecht erhalten zu können, sind regelmäßige Updates ein zentrales Kommunikationsmittel einer Kampagne. Solche Updates stellen die Bemühungen der Initiatoren dar, in denen sie die aktuellen und potenziellen Unterstützer über alle Entwicklungen, die das Projekt betreffen, auf dem Laufenden halten (Mollick 2014). Untersuchungen zeigen, dass die Erfolgsquote für Kampagnen ohne Updates bei 32,6\% und für Kampagnen mit Updates bei $58,7 \%$ liegt (Xu et al. 2014). Updates werden zudem als Signal für Qualität gesehen.

Bezogen auf den Zeitpunkt einer Promotion lässt sich festhalten, dass diese einen Einfluss auf das Ergebnis einer Kampagne haben kann (Lu et al. 2014). Es zeigt sich, dass die Anzahl der Unterstützer und der Umfang der Werbeaktivitäten miteinander korrelieren, jedoch kein signifikanter Nachweis gegeben werden kann, ob diese Aktivitäten sich auch positiv auf den Erfolg der Kampagne auswirken. Ein positiver Effekt auf den Erfolg kann hingegen bei der Zahl der Promoter - Personen, die über soziale Medien von einer Kampagne berichten - gesehen werden. Vor allem zu Beginn der Finanzierungsphase wirkt sich die Zahl der Promoter positiv auf den Kampagnenerfolg aus.

Die richtige Höhe des Fundingziels zu finden ist schwierig, da viele Faktoren einen Einfluss darauf haben. Wird das Fundingziel zu niedrig angesetzt, kann es dazu führen, dass schlussendlich finanzielle Mittel fehlen, um ein Projekt erfolgreich umsetzen zu können. Andersherum kann ein zu hoch gestecktes Fundingziel die Erfolgswahrscheinlichkeit der Kampagne mindern (Mollick 2014). In der Regel ist die Erfolgswahrscheinlichkeit für Kampagnen mit hohen Fundingzielen geringer (Gafni et al. 2018). Insgesamt ist es für die Bestimmung eines angemessenen Fundingziels wichtig, den Kapitalbedarf für die Umsetzung des Projekts gewissenhaft zu kalkulieren. 
Ist das Fundingziel einmal ermittelt und die Kampagne gestartet, wird die Frequenz der Beitragseingänge wichtig. Kommt es nach der ersten Phase der Finanzierung zu einer Stagnation der Zuflüsse verliert das Projekt an Attraktivität. Infolgedessen entscheiden sich die potenziellen Unterstützer gegen eine Investition (Ordanini et al. 2011). Die Projektinitiatoren müssen in der Lage sein, die Dynamik der Finanzierung aufrechtzuerhalten (Ward und Ramachandran 2010).

Als abschließend zu betrachtender Faktor wird die geografische Nähe gesehen. Dieser Faktor definiert die geografische Nähe zwischen dem Initiator und den Unterstützern. Lokale Investoren unterstützen bereits in den frühen Phasen des Finanzierungsprozesses und signalisieren so ihre Verbundenheit mit einem Projekt aus der Region (Agrawal et al. 2013). Hierbei kann eine rein ökonomische Erklärung ausgeschlossen werden (Lin und Viswanathan 2016). Vielmehr sind emotionale Faktoren Treiber solcher Investitionen.

\section{$4 \quad$ Methodisches Vorgehen und Expertenbefragung in Anlehnung an die Delphi-Methode}

\subsection{Die Delphi-Methode als Basis zur Expertenbefragung}

Die methodische Herangehensweise zu dieser Untersuchung basiert auf einer Delphi-Methode. Diese Methode eignet sich für vielfältige Einsatzmöglichkeiten und gibt Raum zur Erfassung unterschiedlicher Gruppenmeinungen (Häder 2014). Sie wird auch als strukturierte, iterativ durchgeführte Gruppenbefragung bezeichnet, welche sich auf dem Wissen und die Prognosefähigkeit von Experten stützt. Ziel ist hierbei auf Basis mehrerer Befragungsrunden zu einem Gruppenurteil zu kommen (Lachmann 1988). Durch das breite Anwendungsgebiet, die Unbestimmtheit und das Fehlen stringenter methodischer Regeln für den Umgang mit Delphi-Befragungen birgt die Delphi-Methode einen großen Gestaltungsspielraum (Häder 2014). Die Delphi-Methode wird in dieser Befragung dazu verwendet, Ansichten einer Expertengruppe über einen „diffusen Sachverhalt“ zu ermitteln. Die Ermittlung unterschiedlicher Expertenmeinungen zu Erfolgsfaktoren des reward-based CF kann als solcher „diffuser Sachverhalt“ angesehen werden. Diese Untersuchung hilft letztendlich dabei, Schlussfolgerungen für erforderliche Interventionen innerhalb des reward-based CF abzuleiten, um dem Problem erfolgloser Kampagnen entgegnen zu können (Häder 2014).

Nach Häder (2014) obliegt die Durchführung einer Delphi-Befragung einzelnen Merkmalen. Darunter zählen die Verwendung eines formalisierten Fragebogens, die Befragung von Experten, die Anonymität der Einzelantworten auf Basis eines standardisierten Frageprogramms, die Ermittlung einer statistischen Gruppenantwort, die Information der Teilnehmer über diese statistische Gruppenantwort sowie eine (mehrfache) Wiederholung der Befragung. Letzteres erfolgt bis zum Erreichen eines vorher festgelegten Abbruchkriteriums wie z. B. das Erreichen einer einheitlichen Gruppenmeinung. 


\subsection{Auswahl der Teilnehmer zur Expertenbefragung}

Duffield (1993) empfiehlt Teilnehmer zu wählen, die über einen notwendigen Einfluss im Untersuchungsfeld verfügen, um letztlich den erzielten Ergebnissen eine praktische Relevanz zuschreiben zu können. Über den Umfang der zu befragenden Expertengruppe herrscht kein einheitliches Bild (Häder 2014). Dabei reichen die Empfehlungen für den Umfang der Expertengruppe von mindestens sieben (Dalkey und Olaf 1963) bis hin zur Empfehlung, eine möglichst große Anzahl von Experten zu befragen (Cuhls et al. 1995).

Die Auswahl der Experten erfolgte mittels Internet- und Literaturrecherche. Um möglichst verschiedene Sichtweisen aufzugreifen, wurden vier zentrale Expertengruppen des reward-based CF definiert: Projektinitiatoren, CF-Berater, Wissenschaftler sowie Projektunterstützer.

Bei der Auswahl der Projektinitiatoren wurde darauf geachtet, dass diese bereits eine erfolgreiche reward-based CF-Kampagne abschließen konnten. Die ausgewählten Unterstützer hingegen, mussten zum Zeitpunkt der Kontaktaufnahme mindestens fünf reward-based CF-Kampagnen unterstützt haben. Dies sollte bei beiden Gruppen die Verfügbarkeit einer gewissen Expertise in diesem Bereich sicherstellen. Die Auswahl der Projektinitiatoren und Unterstützer sowie Kontaktaufnahme erfolgte über frei zugängliche Informationen auf reward-based CF-Plattformen wie Startnext, Kickstarter und Indiegogo.

Die Recherche nach Experten aus der Wissenschaft konzentrierte sich auf die deutschsprachige Literatur. Hierzu wurden entsprechend wissenschaftliche Datenbanken nach themenspezifischen Arbeiten durchsucht. Zusätzlich fand eine Recherche nach Lehrstühlen statt, die im Bereich CF forschen.

Die Expertengruppe der CF-Berater wurde über zur Verfügung stehende Informationen auf eigenen Webseiten sowie über Informationen des German CF Networks kontaktiert.

Auf Basis dieser Expertenrecherche konnten insgesamt 250 potenzielle Experten ermittelt und kontaktiert werden.

Insgesamt konnten auf Basis der Erkenntnisse aus Abschn. 354 Fragen abgefragt werden. Der ausgearbeitete Fragebogen wurde dann auf Konsistenz und Fehlerfreiheit mit fünf Interviewpartnern getestet. Auf Basis der Erkenntnisse der Interviews wurde der Fragebogen entsprechend angepasst. Die Durchführung der Befragung erfolgte über eine webbasierte Plattform. Diese ermöglichte eine zeit- und ortsunabhängige Befragung der Experten. Die Dauer der Befragung lag bei ca. 20 Minuten. Es wurden unterschiedliche Fragetypen gewählt, um ein möglichst ganzheitliches Bild zu Erfolgsfaktoren des reward-based CF ermitteln zu können.

\section{$5 \quad$ Ergebnisse der Expertenbefragung}

\subsection{Stichprobe}

An der ersten Befragungsrunde haben von insgesamt 250 potenziellen Experten insgesamt 66 teilgenommen. Davon haben 62 Experten die Befragung vollständig ausgefüllt, die verbleibenden Experten nur einzelne Fragengruppen. Aus diesem 


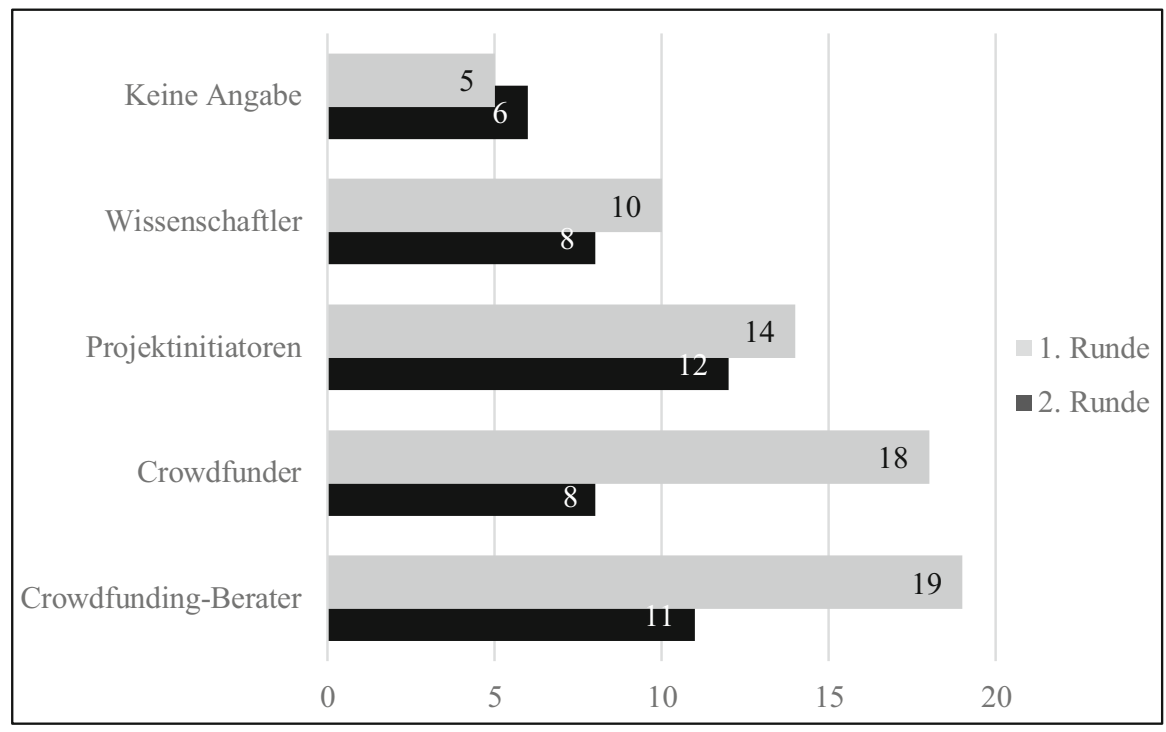

Abb. 1 Gruppierung der Teilnehmer. (Quelle: Eigene Darstellung)

Grund schwankt die Zahl der Experten bei einzelnen Fragengruppen zwischen 62 und 66. Die Expertengruppe gliederte sich in $28 \%$ Prozent weiblicher und 69 \% männlicher Experten; $3 \%$ trafen keine Auswahl. $94 \%$ der Experten gaben an, in der Vergangenheit mindestens eine CF-Kampagne finanziell unterstützt zu haben. Der durchschnittliche Unterstützungsbeitrag dieser lag bei ca. $62 €$.

In der zweiten Befragungsrunde nahmen von den insgesamt 66 Teilnehmern der ersten Runde insgesamt 45 Experten teil. In der zweiten Runde blieb das Geschlechterverhältnis nahezu identisch.

Um möglichst unterschiedliche Sichtweisen in der Expertenbefragung einzubinden, wurden die Experten gebeten, sich einer der jeweiligen Gruppen einzuordnen (Abb. 1).

Zur Sicherstellung der Expertise der Teilnehmer wurden die Teilnehmer gebeten eine Selbsteinschätzung ihrer Expertise für die betrachteten Bereiche vorzunehmen (Tab. 1). Die Expertise der Teilnehmer ist gegeben.

\subsection{Ergebnisse der Expertenbefragung}

Auf Basis der Expertenbefragung konnten für die definierten drei unterschiedlichen Bereiche Ergebnisse ermittelt werden. Diese Ergebnisse werden im Folgenden näher erläutert.

\subsubsection{Personenspezifische Erfolgsfaktoren}

Die Mehrheit der Befragten sieht die Größe des sozialen Netzwerks eines Projektinitiators als wichtigen Einflussfaktor zum erfolgreichen Kampagnenabschluss (82 \%) 


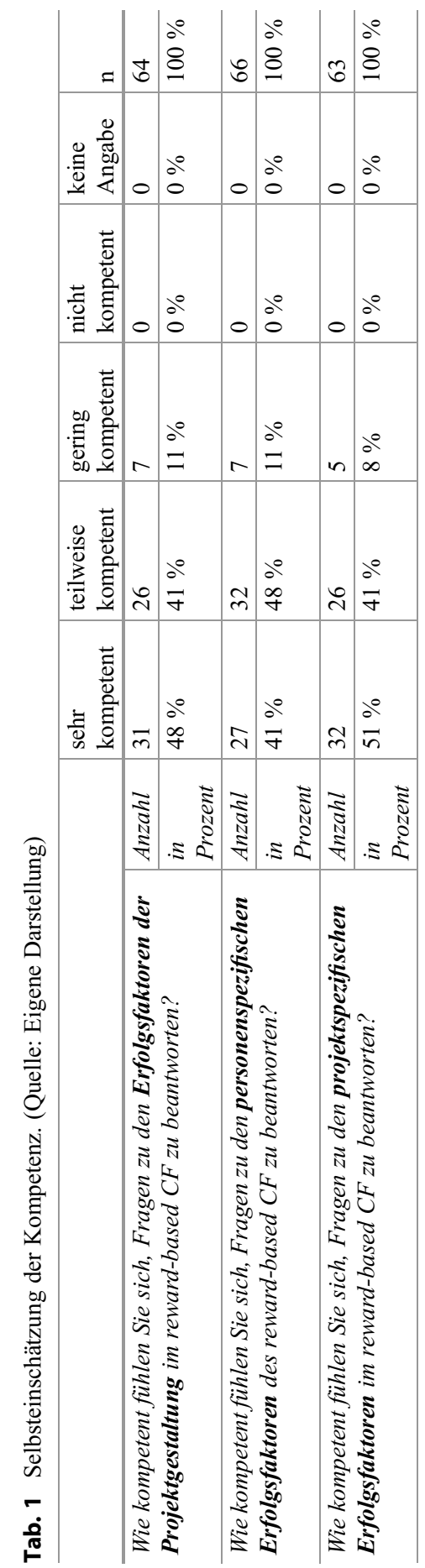


(Tab. 2). Rund $70 \%$ der Befragten ist zudem der Meinung, dass im Vorfeld erfolgreich abgeschlossene CF-Kampagnen die Erfolgswahrscheinlichkeit einer anstehenden Kampagne erhöhen. Die aktive Kommunikation mit der Crowd ist nach Meinung der Experten ein sehr wichtiges Mittel, um die Erfolgschance einer Kampagne zu erhöhen. Dies schlägt sich auch mit einer hohen Zustimmung von $72 \%$ nieder. Die Frage zur Wichtigkeit des Bildungsstands des Initiators kann nicht ausreichend geklärt werden. Nur $32 \%$ der Befragten sehen diesen Aspekt als bedeutend zur Steigerung der Unterstützungsbereitschaft der Crowd an; $26 \%$ stimmen diesem Aspekt eher nicht zu. Für $30 \%$ nimmt die Unterstützungshistorie des Initiators eher keinen Einfluss auf die Erfolgschancen.

Zum Abschluss des Fragenblocks der ersten Umfragerunde wurden die Teilnehmer gebeten, weitere mögliche personenspezifische Erfolgsfaktoren zu benennen. Dieser Aspekt wurde dann in der zweiten Runde abgefragt. Als möglicher Erfolgsfaktor wurde aufgeführt, dass eine extrovertierte Persönlichkeit eines Projektinitiators zu höheren Erfolgschancen führt. Das Ergebnis aus der zweiten Befragungsrunde zeigt, dass $38 \%$ der Befragten diesem Aspekt eher zustimmen, während die Mehrheit von $42 \%$ bei dieser Frage unentschlossen bleibt.

Abschließend wurden die Befragten gebeten, die entsprechenden personenspezifischen Erfolgsfaktoren hinsichtlich ihrer Wichtigkeit zu ordnen (Tab. 3). Hierbei wurde die Kommunikationsstärke neben der Größe des sozialen Netzwerks als zentraler Erfolgsfaktor gesehen.

\subsubsection{Erfolgsfaktoren der Projektgestaltung}

Der allgemeinen Frage nach der Wichtigkeit der Projektgestaltung stimmten $95 \%$ der Teilnehmer zu (Abb. 2). Somit sehen die Teilnehmer diesen Aspekt als einen Kernpunkt innerhalb einer Kampagnengestaltung an. Zur näheren Beleuchtung dieses Aspektes wurden weitere Details abgefragte, die auf eine gelungene Projektbeschreibung abzielen. Die Mehrheit der Teilnehmer findet, dass eine gelungene Projektbeschreibung Begeisterung für ein Projekt erwecken sollte. Zudem sehen $23 \%$ der Befragten eine persönliche und authentische Wirkung als wichtig an. Darüberhinaus sollte für $21 \%$ der Befragten eine gelungene Projektbeschreibung klar und verständlich formuliert sein. Die Transparenz des Verwendungszwecks der Gelder sehen $19 \%$ als wichtig an. Weniger wichtig erscheint dabei mit $4 \%$ die Kreativität, mit $3 \%$ die Fehlerfreiheit und mit $2 \%$ die Kürze und Prägnanz der enthaltenen Antworten.

Die Erkenntnisse des E-Commerce, dass eine Projektpräsentation sowohl lebendig als auch eine hohe soziale Präsenz aufweisen sollte, wird von einem Großteil der Befragten für das reward-based CF bestätigt (Tab. 4). Ein nicht ganz so klares Bild, ergibt sich zu der Aussage, dass die Präsentation (Projektseite) einer Kampagne interaktiv sein sollte. Hier stimmen nur $14 \%$ bzw. $31 \%$ zu. In der zweiten Befragungsrunde stieg die Zahl der zustimmenden Experten auf $40 \%$. In der zweiten Befragungsrunde wurde das zunächst gewählte Beispiel überarbeitet, um das Verständnis der Experten zu erhöhen. Der Einsatz von Bildern und Grafiken gegenüber Texten ist laut der Mehrheit (38 \%) der Befragten zu bevorzugen. Darüberhinaus sind sich die Befragten einig, dass Kampagnen mit einem Pitch-Video höhere 


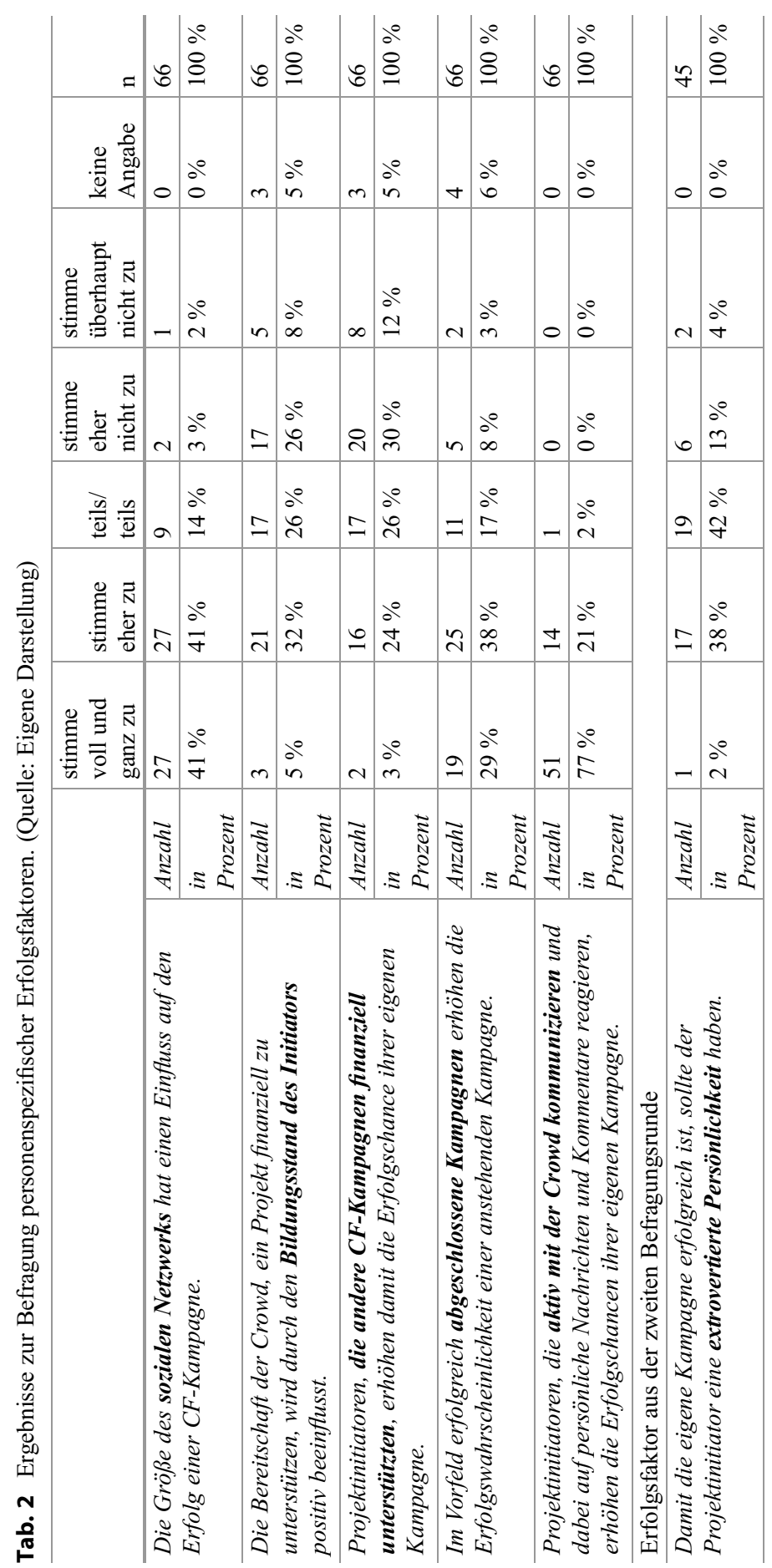


Tab. 3 Rangordnung personenspezifischer Erfolgsfaktoren. (Quelle: Eigene Darstellung)

\begin{tabular}{|c|c|c|c|c|c|}
\hline Personenspezifische Erfolgsfaktoren & 1. & 2. & 3. & 4. & 5 \\
\hline Größe des sozialen Netzwerks & $22,58 \%$ & $38,71 \%$ & $25,81 \%$ & $8,06 \%$ & $4,84 \%$ \\
\hline $\begin{array}{l}\text { Bildungsstand des Initiators/ } \\
\text { Projektteams }\end{array}$ & $6,45 \%$ & $6,45 \%$ & $6,45 \%$ & $32,26 \%$ & $48,39 \%$ \\
\hline Unterstützungshistorie & $1,61 \%$ & $6,45 \%$ & $22,58 \%$ & $38,71 \%$ & $30,65 \%$ \\
\hline Vergangene Erfolge im CF & $9,68 \%$ & $17,74 \%$ & $41,94 \%$ & $19,35 \%$ & $11,29 \%$ \\
\hline $\begin{array}{l}\text { Kommunikationsstärke des } \\
\text { Initiators/Projektteams }\end{array}$ & $59,68 \%$ & $30,65 \%$ & $3,23 \%$ & $1,61 \%$ & $4,84 \%$ \\
\hline
\end{tabular}

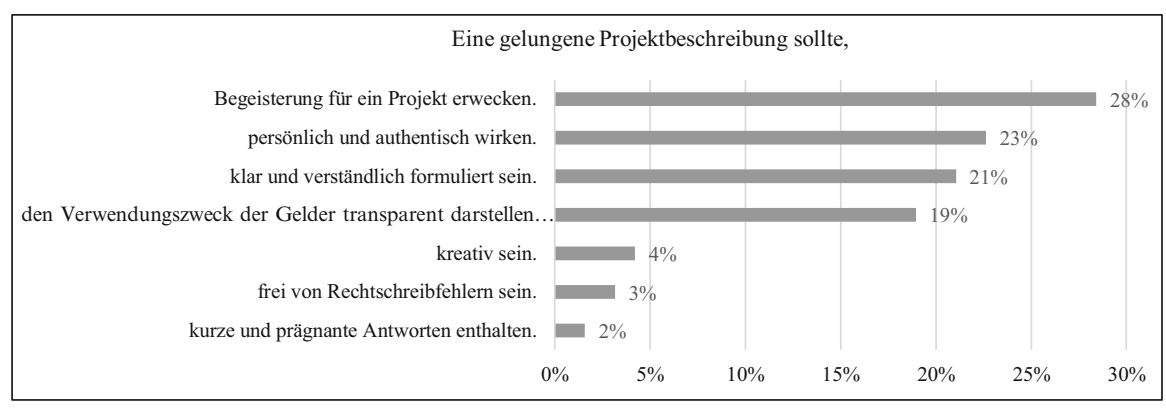

Abb. 2 Inhalte einer Projektbeschreibung. (Quelle: Eigene Darstellung)

Fundingsummen erzielen als Kampagnen ohne Pitch-Video. $72 \%$ stimmen dem voll und ganz $\mathrm{zu}$.

Gemäß der Rangfolgendefinition der Experten sollte ein Pitch-Video in den ersten Sekunden gezielt über das Projektvorhaben aufklären (Tab. 5). Eine authentische Darstellung ist darüberhinaus von Bedeutung, bei der sich der Initiator und das Projektteam dem potentiellen Unterstützer präsentieren. Die Videolänge belegt den 8. Rang. Als minder wichtig sehen die Experten das Budget für die Produktion des Pitch-Videos. Demnach spielen für die Experten Kosten eines Videos kaum eine Rolle. Somit können auch einfach produzierte Videos Verwendung finden, sofern diese andere Kriterien erfüllen. Für Unterstützer können die Videos eine Länge von ca. 4,66 Minuten haben, während die Berater zu 2,47 Minuten tendieren (Tab. 6). Im Mittel liegt die anzustrebende Dauer bei 3,64 Minuten, was einer Dauer von rund 3 Minuten und 38 Sekunden entspricht.

Abschließend wurden die Befragten gebeten, die entsprechenden Erfolgsfaktoren der Projektgestaltung hinsichtlich ihrer Wichtigkeit zu ordnen (Tab. 7). Hierbei wurde der Projektbeschreibung und dem Pitch-Video der höchste Rang zugeordnet.

\subsubsection{Projektspezifische Erfolgsfaktoren}

Die Mehrheit der Experten (79 \%) sehen eine innovative Projektidee, die der Crowd einen klaren Mehrwert liefert und sich von anderen Projekten abhebt, ausschlaggebend für den Erfolg einer Kampagne (Tab. 8). Die Exklusivität von Gegenleistungen wird ebenfalls als wichtiger Aspekt einer erfolgreichen Kampagne gesehen (76 \%). 


\begin{tabular}{|c|c|c|c|c|c|c|c|c|c|c|c|c|}
\hline$\Rightarrow$ & 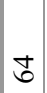 & $\begin{array}{l}0 \\
8 \\
0\end{array}$ & f & $\begin{array}{l}\circ \\
\stackrel{0}{0}\end{array}$ & do & $\begin{array}{l}0 \\
\stackrel{0}{0}\end{array}$ & t & $\begin{array}{l}0 \\
8 \\
0\end{array}$ & J & $\begin{array}{l}{ }^{\circ} \\
\stackrel{8}{0}\end{array}$ & t & $\begin{array}{l}0^{0} \\
8 \\
0\end{array}$ \\
\hline 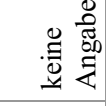 & - & $\begin{array}{l}a^{\circ} \\
i\end{array}$ & 0 & $\partial^{\circ}$ & - & $\begin{array}{l}0 \\
\mathrm{n}\end{array}$ & 0 & $\partial^{\circ}$ & - & $\begin{array}{l}\stackrel{0}{ } \\
i\end{array}$ & - & $\begin{array}{l}0^{\circ} \\
\mathrm{N}\end{array}$ \\
\hline 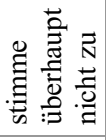 & $\nabla$ & $\begin{array}{l}0^{\circ} \\
0\end{array}$ & $v$ & $\begin{array}{l}\partial 0 \\
\dot{\theta}\end{array}$ & 0 & $\partial_{0}^{\circ}$ & - & $\begin{array}{l}\partial^{\circ} \\
\text { i }\end{array}$ & 0 & $\begin{array}{l}0^{\circ} \\
0\end{array}$ & - & $\begin{array}{l}\partial^{\circ} \\
\text { i }\end{array}$ \\
\hline 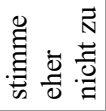 & 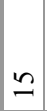 & $\begin{array}{l}\partial^{\circ} \\
\text { ते }\end{array}$ & $m$ & $a^{\circ}$ & 0 & $\partial^{\circ}$ & $N$ & $\begin{array}{l}\partial^{\circ} \\
m\end{array}$ & $m$ & $\begin{array}{l}0^{\circ} \\
\text { in }\end{array}$ & - & $\partial^{\circ}$ \\
\hline$\frac{\bar{a}}{\bar{\theta}} \frac{n}{\bar{\theta}}$ & $\because$ & $\begin{array}{l}\partial^{0} \\
\hat{\imath}\end{array}$ & $a$ & $\begin{array}{l}0^{\circ} \\
\stackrel{i}{ }\end{array}$ & in & $\begin{array}{l}0^{0} \\
\infty\end{array}$ & $\nabla$ & do & 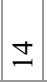 & 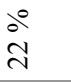 & in & $\begin{array}{l}0^{0} \\
\infty\end{array}$ \\
\hline 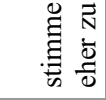 & 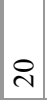 & $\frac{\partial^{\circ}}{m}$ & $\stackrel{\infty}{-}$ & $\dot{0}^{\circ}$ & त̃ & $\begin{array}{l}0^{\circ} \\
\text { m }\end{array}$ & $\stackrel{d}{\sim}$ & $\begin{array}{l}\delta^{0} \\
\infty \\
m\end{array}$ & त̂ & $\begin{array}{l}\text { dீ } \\
\text { iे }\end{array}$ & $\cong$ & $\begin{array}{l}0^{\circ} \\
0\end{array}$ \\
\hline 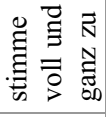 & $a$ & $\begin{array}{l}\partial^{\circ} \\
\pm\end{array}$ & $\cong$ & $\begin{array}{l}\partial^{\circ} \\
\stackrel{2}{2}\end{array}$ & m & $\begin{array}{l}\delta^{\circ} \\
i \\
\wp\end{array}$ & $m$ & $\begin{array}{l}\partial^{\circ} \\
i n\end{array}$ & $\vec{d}$ & $\begin{array}{l}0^{\circ} \\
\infty \\
\infty\end{array}$ & fo & $\begin{array}{l}\AA^{0} \\
i\end{array}$ \\
\hline & 齐 & 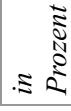 & \begin{tabular}{l}
$\mathcal{Z}$ \\
\multirow{2}{*}{} \\
$\mathcal{1}$
\end{tabular} & 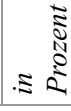 & 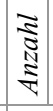 & 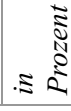 & 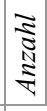 & 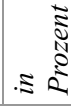 & $\mid$ & 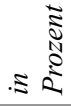 & 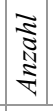 & $\approx$ ह \\
\hline & 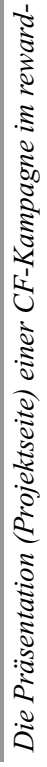 & & is & & 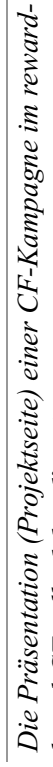 & & 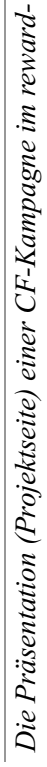 & 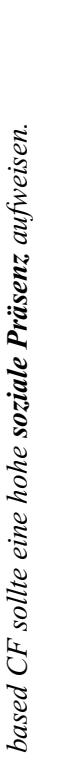 & 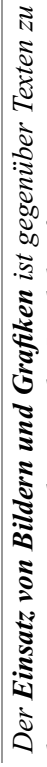 & 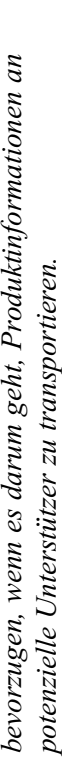 & 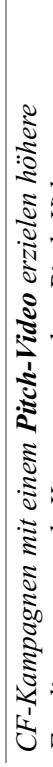 & 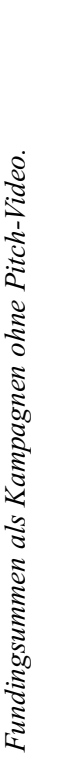 \\
\hline
\end{tabular}




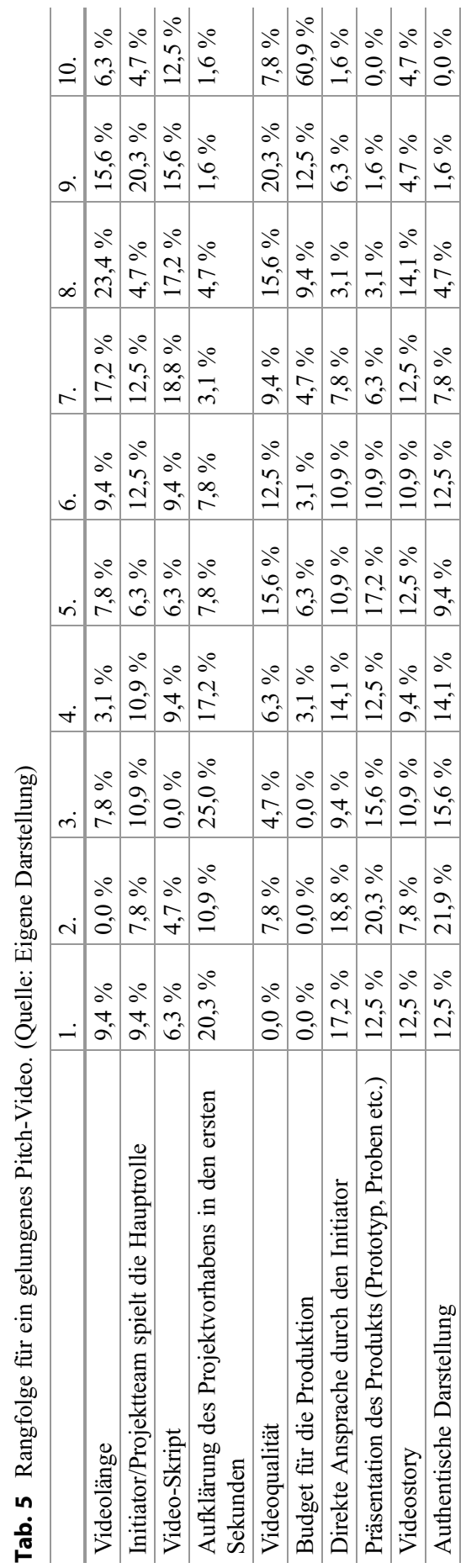


Tab. 6 Gruppenvergleich Dauer des Pitch-Videos. (Quelle: Eigene Darstellung)

\begin{tabular}{l|l|l|l}
\hline Gruppe & Mittelwert & N & Std. Abw. \\
\hline Projektinitiatoren & 3,68 Minuten & 14 & 0,95 Minuten \\
\hline Berater & 2,47 Minuten & 19 & 0,72 Minuten \\
\hline Crowdfunder & 4,66 Minuten & 18 & 1,68 Minuten \\
\hline Forscher & 4,15 Minuten & 10 & 2,33 Minuten \\
\hline
\end{tabular}

Tab. 7 Rangordnung: Erfolgsfaktoren der Projektgestaltung. (Quelle: Eigene Darstellung)

\begin{tabular}{|c|c|c|c|c|c|c|c|c|}
\hline $\begin{array}{l}\text { Erfolgsfaktoren der } \\
\text { Projektgestaltung }\end{array}$ & 1. & 2. & 3. & 4. & 5. & 6. & 7. & 8. \\
\hline Projektbeschreibung & $41,9 \%$ & $16,1 \%$ & $11,3 \%$ & $6,5 \%$ & $12,9 \%$ & $4,8 \%$ & $1,6 \%$ & $4,8 \%$ \\
\hline $\begin{array}{l}\text { Interaktivität der } \\
\text { Projektseite }\end{array}$ & $3,2 \%$ & $8,1 \%$ & $9,7 \%$ & $4,8 \%$ & $11,3 \%$ & $14,5 \%$ & $24,2 \%$ & $24,2 \%$ \\
\hline $\begin{array}{l}\text { Lebendigkeit der } \\
\text { Projektseite }\end{array}$ & $8,1 \%$ & $24,2 \%$ & $12,9 \%$ & $16,1 \%$ & $19,4 \%$ & $14,5 \%$ & $4,8 \%$ & $0,0 \%$ \\
\hline $\begin{array}{l}\text { Soziale Präsenz der } \\
\text { Projektseite }\end{array}$ & $12,9 \%$ & $11,3 \%$ & $19,4 \%$ & $25,8 \%$ & $4,8 \%$ & $14,5 \%$ & $4,8 \%$ & $6,5 \%$ \\
\hline $\begin{array}{l}\text { Einsatz von Bildern } \\
\text { \& Grafiken }\end{array}$ & $3,2 \%$ & $9,7 \%$ & $21,0 \%$ & $21,0 \%$ & $22,6 \%$ & $14,5 \%$ & $8,1 \%$ & $0,0 \%$ \\
\hline Pitch-Video & $29,0 \%$ & $16,1 \%$ & $19,4 \%$ & $11,3 \%$ & $9,7 \%$ & $4,8 \%$ & $6,5 \%$ & $3,2 \%$ \\
\hline $\begin{array}{l}\text { Dauer des Pitch- } \\
\text { Videos }\end{array}$ & $0,0 \%$ & $12,9 \%$ & $6,5 \%$ & $3,2 \%$ & $6,5 \%$ & $12,9 \%$ & $21,0 \%$ & $37,1 \%$ \\
\hline
\end{tabular}

Bei der Frage "Staffelung der Unterstützungsbeiträge mit niedrigen Werten von unter $15 €$ beginnend, erhöht die Erfolgschancen", ist die Eindeutigkeit nicht gegeben. Dem entgegen steht jedoch die allgemeine Betrachtung der Staffelung von Unterstützungsbeiträgen. Gemäß $81 \%$ der Befragten ist dieser Aspekt als wichtig zu betrachten. Die Mehrheit der Befragten (56 \%) sieht im Aufbau einer Community positive Auswirkungen auf die Anzahl der Unterstützer und die Höhe der erzielten Fundingsumme. Laut 37 \% der Experten nimmt die Unterstützergruppe „Familie und Freunde“ einen starken Einfluss auf die Bekanntmachung einer CF-Kampagne. Die geografische Nähe zwischen Initiator und dem Unterstützer wird nicht als zentraler Aspekt bestätigt.

Die Anzahl der Unterstützer, die im ersten Drittel der Finanzierungsphase gewonnen werden konnten, beeinflusst nach Meinung der Befragten den weiteren Verlauf der Kampagne positiv. Insgesamt stimmen dem $87 \%$ zu. Hiermit eng verbunden ist der zentrale Aspekt sozialer Netzwerke. Die Bedeutung dieser wurde innerhalb der Befragung bestätigt. So sehen $71 \%$, dass sich die Verknüpfung der Kampagne mit sozialen Netzwerken positiv auf den Erfolg dieser auswirkt (Tab. 8). Darüberhinaus ist die Verknüpfung der CF-Kampagne mit einer Facebook-Fanseite mit $81 \%$ am erfolgversprechendsten (Abb. 3). Nur $17 \%$ sehen die Verknüpfung der Kampagne mit dem persönlichen Facebook-Profil des Initiators als erfolgversprechend an. Twitter als soziales Medium spielt in dieser Befragung keine Rolle. 


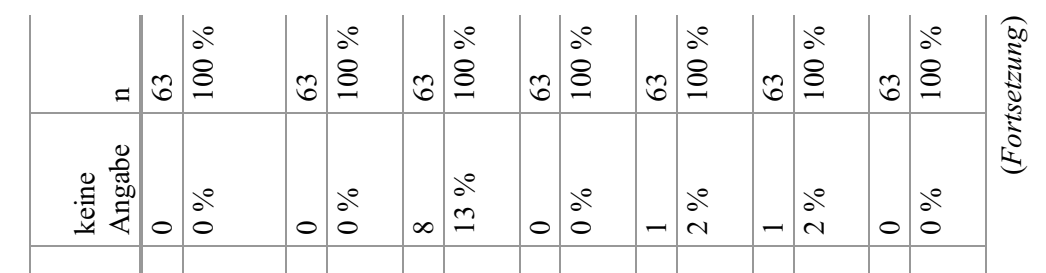

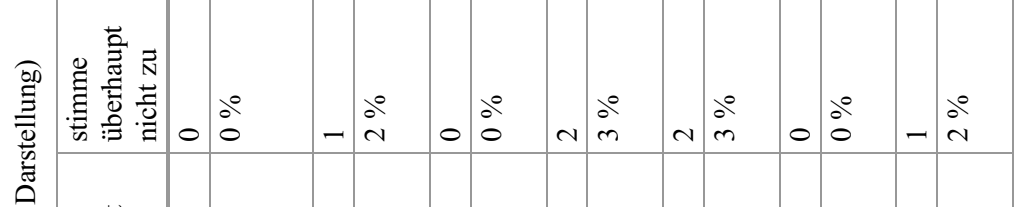

兽

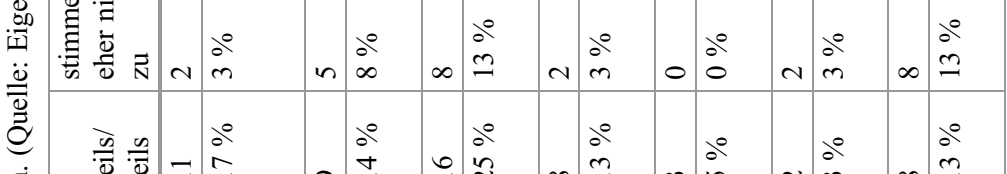

\begin{tabular}{|c|c|c|c|c|c|c|c|c|c|c|c|c|}
\hline$\frac{\bar{w}}{\bar{D}} \frac{n}{\bar{\theta}}$ & $=$ & = & $a$ & $\begin{array}{l}0^{\circ} \\
\pm\end{array}$ & 0 & $i$ & $\infty$ & $\begin{array}{l}0^{\circ} \\
2\end{array}$ & $m$ in & $\sim \mid \begin{array}{l}a^{\circ} \\
m\end{array}$ & $\infty$ & $\begin{array}{l}0^{\circ} \\
2\end{array}$ \\
\hline 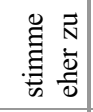 & $\stackrel{\sim}{\sim}$ & $\frac{\partial^{\circ}}{7}$ & $\stackrel{\infty}{\sim}$ & $\begin{array}{l}\partial^{\circ} \\
\dot{y}\end{array}$ & $\ddot{\imath}$ & $\begin{array}{l}a^{\circ} \\
\text { n }\end{array}$ & $m$ & $\begin{array}{l}i^{\circ} \\
\text { in }\end{array}$ & $\simeq a^{\circ}$ & $\approx \frac{d^{\circ}}{i}$ & $\approx$ & $\begin{array}{l}a^{\circ} \\
\text { n }\end{array}$ \\
\hline 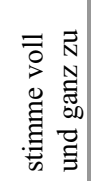 & $\stackrel{ \pm}{\sim}$ & $\begin{array}{l}{ }^{\circ} \\
\infty \\
m\end{array}$ & 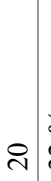 & $\begin{array}{l}\dot{\sigma}^{\circ} \\
\text { ñ }\end{array}$ & 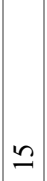 & $\begin{array}{l}\stackrel{2}{\sim} \\
\stackrel{\Delta}{d}\end{array}$ & $\infty$ & ڤे & $\stackrel{2}{q} \frac{\partial}{\pi}$ & 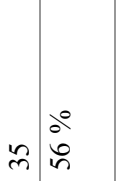 & $\hat{\imath}$ & $\begin{array}{l}a^{\circ} \\
\text { in }\end{array}$ \\
\hline & 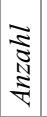 & 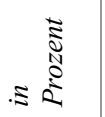 & 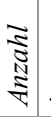 & $\approx$ ह & 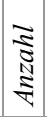 & $\approx$ ह & 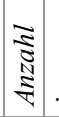 & 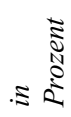 & 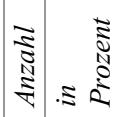 & 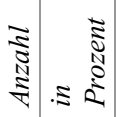 & 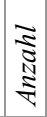 & 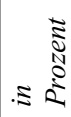 \\
\hline & 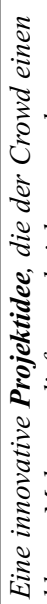 & 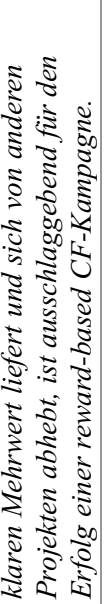 & 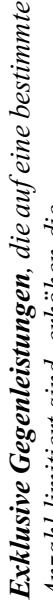 & 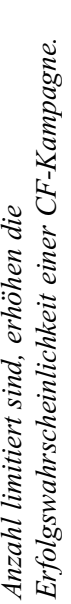 & & 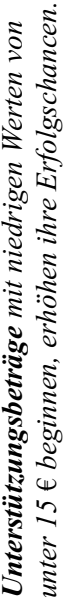 & 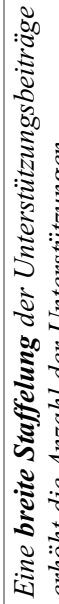 & 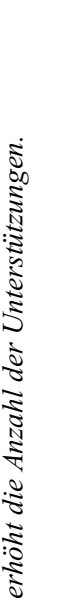 & 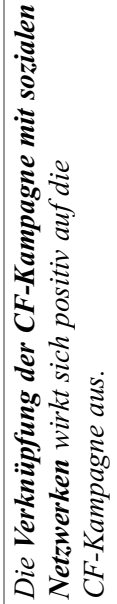 & 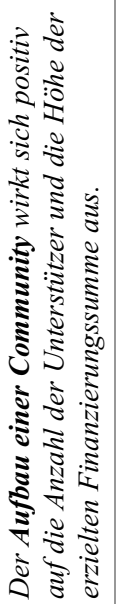 & 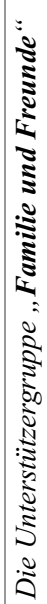 & 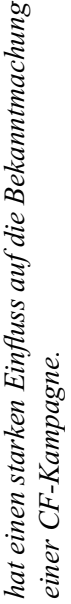 \\
\hline
\end{tabular}




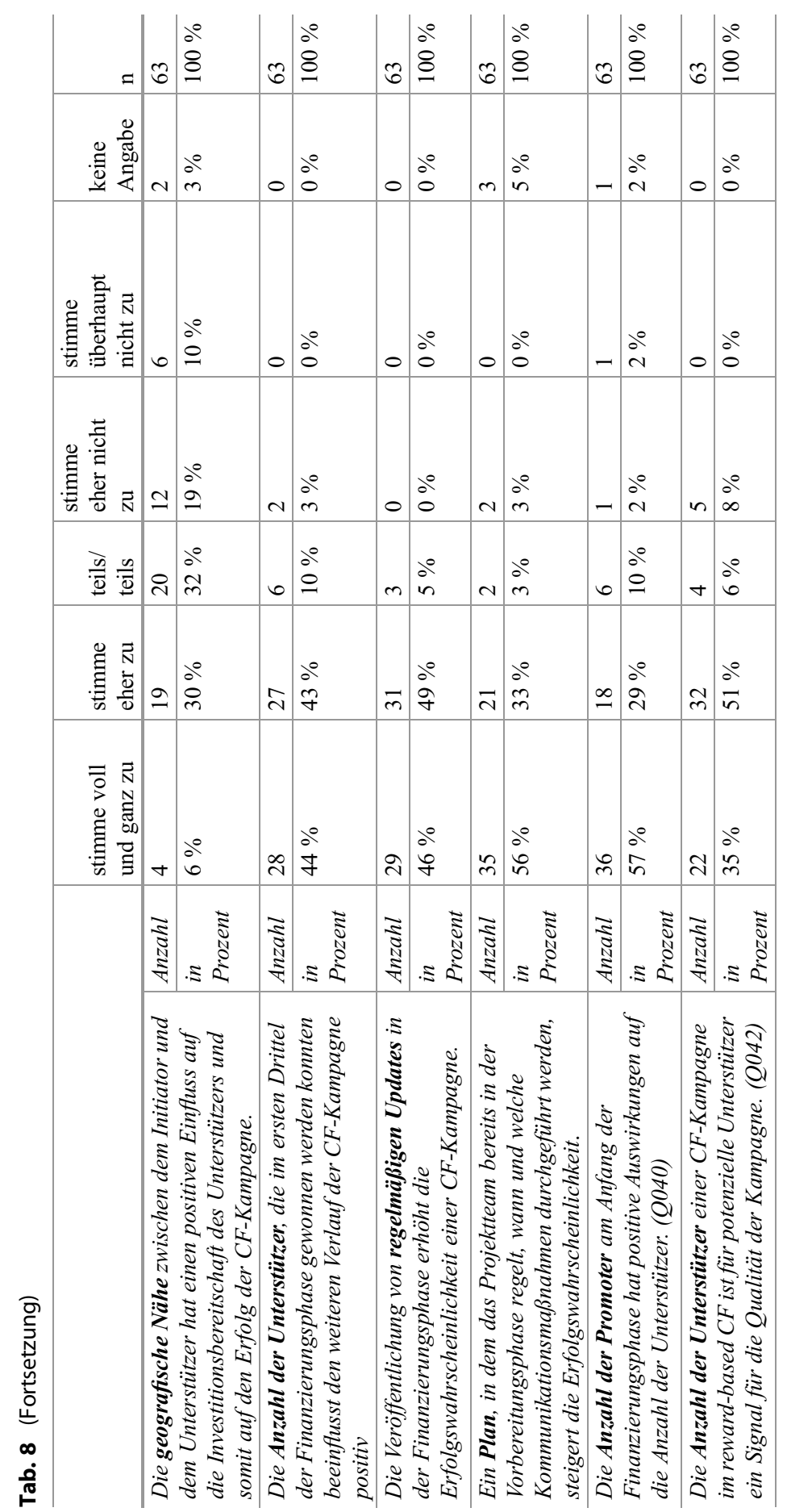


Abb. 3 Die Verknüpfung mit sozialen Netzwerken. (Quelle: Eigene Darstellung)

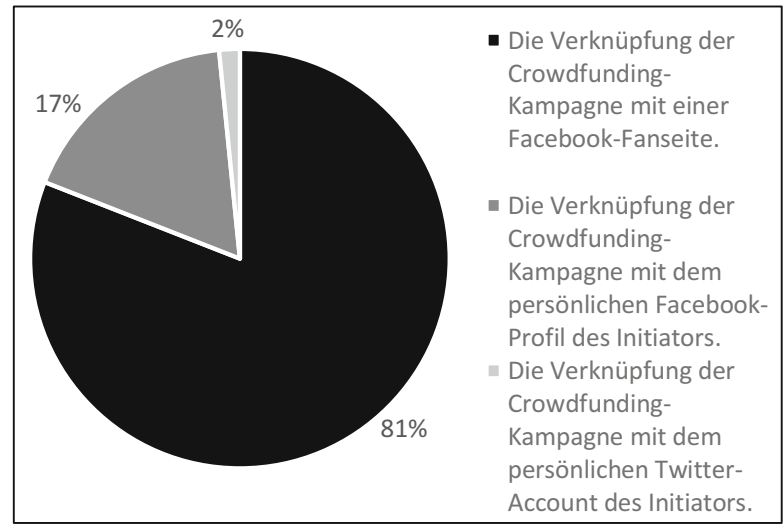

Tab. 9 Nutzung von Promotionskanäle unter Kosten-Nutzen Gesichtspunkten. (Quelle: Eigene Darstellung)

\begin{tabular}{l|l|l|l|l}
\hline & häufig & gelegentlich & gar nicht & keine Angabe \\
\hline Soziale Netzwerke & $96,8 \%$ & $0,0 \%$ & $1,6 \%$ & $1,6 \%$ \\
\hline TV & $0,0 \%$ & $36,5 \%$ & $57,1 \%$ & $6,4 \%$ \\
\hline Radio & $7,9 \%$ & $63,5 \%$ & $25,4 \%$ & $3,2 \%$ \\
\hline Presse & $50,8 \%$ & $49,2 \%$ & $0,0 \%$ & $0,0 \%$ \\
\hline Mlyer & $9,5 \%$ & $57,1 \%$ & $25,4 \%$ & $7,9 \%$ \\
\hline E-Mail & $93,7 \%$ & $6,4 \%$ & $0,0 \%$ & $0,0 \%$ \\
\hline Blogs & $50,8 \%$ & $47,6 \%$ & $1,6 \%$ & $0,0 \%$ \\
\hline Messen & $77,8 \%$ & $17,5 \%$ & $1,6 \%$ & $3,2 \%$ \\
\hline
\end{tabular}

Laut $57 \%$ der Befragten hat die Anzahl der Promoter am Anfang der Finanzierungsphase positive Auswirkungen auf die Anzahl der Unterstützer (Tab. 8). Und $35 \%$ der Befragten sehen die Anzahl der Unterstützer als ein Signal für die Qualität der Kampagne an.

$56 \%$ der Befragten stimmen der Aussage zu, dass ein Kommunikationsplan, in dem das Projektteam bereits in der Vorbereitungsphase regelt, wann und welche Kommunikationsmaßnahmen durchgeführt werden, die Erfolgschancen steigert (Tab. 8). Dabei stellen die Experten heraus, dass im Mittel 24,47 Tage vor Beginn der Finanzierungsphase mit den Marketingmaßnahmen begonnen werden. Deutlich wird zudem, dass soziale Netzwerke und Mund-zu-Mund-Propaganda, unter den Befragten eine sehr wichtige Rolle spielen (Tab. 9).

Die Nutzung von sozialen Netzwerken ermöglicht es Initiatoren, von verschiedenen Vorteilen zu profitieren. Die Vorteile gemäß der Aussage der Experten liegen vor allem in der direkten Kommunikation mit der Crowd und der schnellen und unkomplizierten Informationsausbreitung (Tab. 10). 


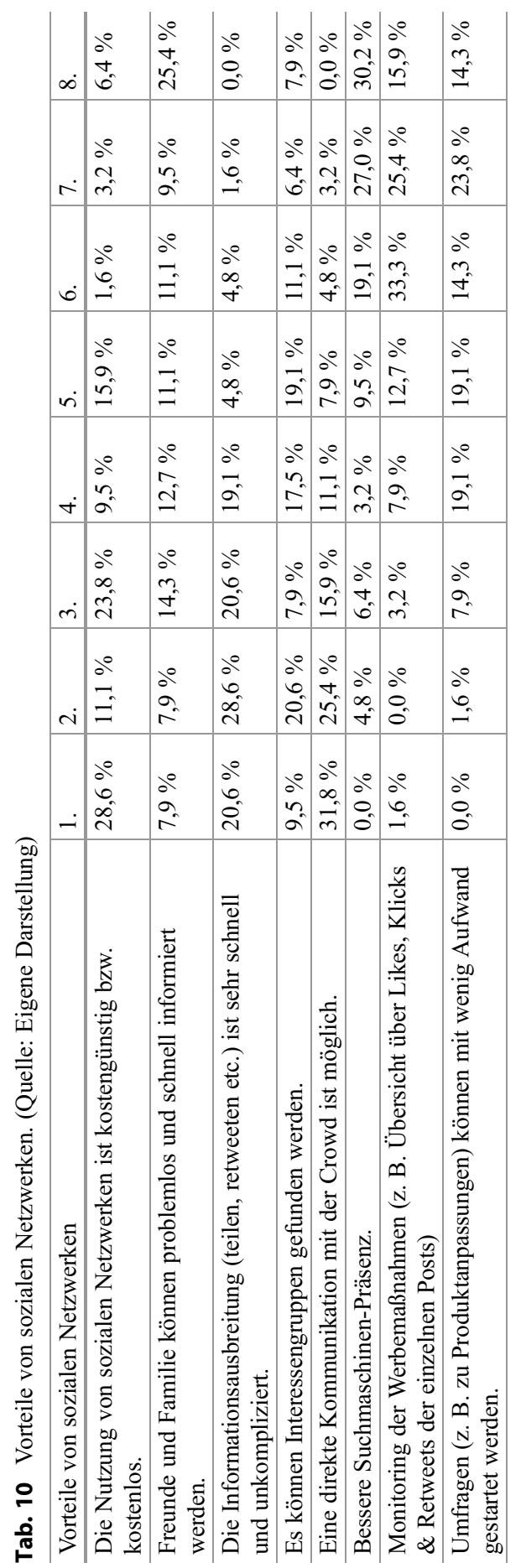




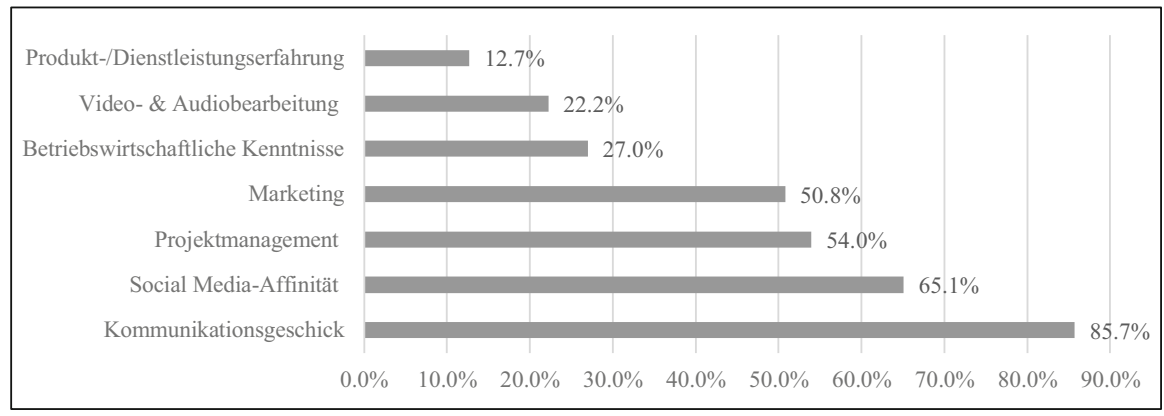

Abb. 4 Erfolgreicher Projektabschluss: Erfahrungen, Kenntnisse und Fähigkeiten. (Quelle: Eigene Darstellung)

Tab. 11 Gruppenvergleich: Dauer der CF-Kampagne. (Quelle: Eigene Darstellung)

\begin{tabular}{l|l|l|l}
\hline Gruppe & Mittelwert & N & Std. Abweichung \\
\hline Projektinitiatoren & 45,7 Tage & 14 & 15,67 Tage \\
\hline Berater & 46,3 Tage & 19 & 18,42 Tage \\
\hline Unterstützer & 55 Tage & 18 & 39,83 Tage \\
\hline Forscher & 38 Tage & 10 & 16,41 Tage \\
\hline
\end{tabular}

Tab. 12 Gruppenvergleich: Anzahl Updates in der Finanzierungsphase. (Quelle: Eigene Darstellung)

\begin{tabular}{l|l|l|l|l|l|l}
\hline Gruppe & Mittelwert & Min. & Max. & Std. Abw. & Modus & N \\
\hline Projektinitiatoren & 6,3 Updates & 2 Updates & 10 Updates & 2,87 Updates & 10 & 14 \\
\hline Berater & 7,5 Updates & 3 Updates & 25 Updates & 5,84 Updates & $3 ; 5$ & 19 \\
\hline Crowdfunder & 5,2 Updates & 3 Updates & 15 Updates & 2,91 Updates & 5 & 18 \\
\hline Forscher & 5,7 Updates & 2 Updates & 12 Updates & 3,33 Updates & 3 & 10 \\
\hline
\end{tabular}

Erfahrungen, Kenntnisse und Fähigkeiten eines Projektinitiators sind ein wichtiger Faktor zur Erreichung des Finanzierungsziels. So wird vor allem das Kommunikationsgeschick $(85,7 \%)$ sowie Social Media-Affinität $(65,1 \%)$ als bedeutend angesehen (Abb. 4).

Die Dauer einer Crowdfundingkampagne sollte im Durchschnitt nicht länger als 47,7 Tage sein und sich im Zeitraum von 30 bzw. 45 Tagen bewegen. Auffällig beim Vergleich der einzelnen Expertengruppen ist, dass zum einen die vorgeschlagenen Tage der Projektinitiatoren und Berater sehr nah beieinander liegen, während die Angabe der Forscher deutlich unter der Angabe der Unterstützer liegt (Tab. 11).

Umsetzung regelmäßiger Updates in der Finanzierungsphase ist für den Großteil der Befragten ein deutliches Mittel, um die Erfolgswahrscheinlichkeit zu erhöhen. Insgesamt sehen $95 \%$ der Befragten diesen Aspekt als relevant an. Dabei lag die Zahl der Umsetzung von Updates im Durchschnitt bei 6,3 Updates (Tab. 12). Insgesamt schlagen alle Experten mindestens 2-3 Updates in der Finanzierungsphase vor. 
Tab. 13 Vorteile der Teamarbeit im reward-based CF. (Quelle: Eigene Darstellung)

\begin{tabular}{l|l|l|l|l|l|l}
\hline Vorteile der Teamarbeit & 1. & 2. & 3. & 4. & 5. & 6. \\
\hline $\begin{array}{l}\text { Ein Projektteam bietet ein } \\
\text { größeres soziales Netzwerk. }\end{array}$ & $34,9 \%$ & $9,5 \%$ & $17,5 \%$ & $19,1 \%$ & $7,9 \%$ & $11,1 \%$ \\
\hline $\begin{array}{l}\text { Ein Projektteam hat einen } \\
\text { größeren Erfahrungsschatz. }\end{array}$ & $19,1 \%$ & $36,5 \%$ & $23,8 \%$ & $11,1 \%$ & $6,4 \%$ & $3,2 \%$ \\
\hline $\begin{array}{l}\text { Ein Projektteam wird als } \\
\text { vertrauenswürdiger } \\
\text { wahrgenommen als ein } \\
\text { einzelner Initiator (Vier-Augen- } \\
\text { Prinzip). }\end{array}$ & $14,3 \%$ & $9,5 \%$ & $12,7 \%$ & $22,2 \%$ & $33,3 \%$ & $7,9 \%$ \\
\hline $\begin{array}{l}\text { Durch Arbeitsteilung können } \\
\text { sich die Teammitglieder } \\
\text { thematisch spezialisieren. }\end{array}$ & $14,3 \%$ & $30,2 \%$ & $23,8 \%$ & $19,1 \%$ & $9,5 \%$ & $3,2 \%$ \\
\hline $\begin{array}{l}\text { Ein Projektteam erzeugt bessere } \\
\text { Arbeitsergebnisse. }\end{array}$ & $12,7 \%$ & $7,9 \%$ & $11,1 \%$ & $23,8 \%$ & $31,8 \%$ & $12,7 \%$ \\
\hline $\begin{array}{l}\text { Ein Projektteam erreicht höhere } \\
\text { Fundingsummen. }\end{array}$ & $4,8 \%$ & $6,4 \%$ & $11,1 \%$ & $4,8 \%$ & $11,1 \%$ & $61,9 \%$ \\
\hline
\end{tabular}

Die Arbeit in Teams bietet für eine Kampagne unterschiedliche Vorteile. Der größte Nutzen aus einer gemeinschaftlichen Kampagne liegt in der Größe des zur Verfügung stehenden sozialen Netzwerks (Tab. 13).

Als Qualitätssignal wurde die Einsatzbereitschaft des Initiators bzw. des Projektteams definiert. Gemäß Expertenbefragung können Initiatoren die Einsatzbereitschaft vor allem in der authentischen Darstellung des Projekts und der Idee sowie der direkten Kommunikation mit der Crowd und der schnellen Beantwortung von Fragen sowie der Veröffentlichung von regelmäßigen Updates signalisieren (Tab. 14).

Eine ausgereifte Projektplanung wirkt sich nach Meinung der Befragten (56\%) positiv auf die erfolgreiche Auslieferung der Gegenleistung aus (Tab. 15). In diesem Zusammenhang wurden Gründe ermittelt, die zu einer verspäteten Auslieferung von Gegenleistungen führen können und somit einer ausgereiften Projektplanung entgegenstehen (Abb. 5). Hierzu zählen vor allem die fehlende Erfahrung des Projektteams $(42,86 \%)$ und eine ungenaue Planung $(31,16 \%)$.

Das Fundingziel höher anzusetzen als die tatsächlich benötigte Summe ist für die Befragten kein triftiger Grund, der die Erfolgschancen der Kampagne senken könnte (Tab. 15). Um einen tieferen Einblick darüber zu gewinnen, inwieweit das angegebene Fundingziel die tatsächlich benötigte Summe übersteigen darf, wurde folgende Frage in der zweiten Befragungsrunde gestellt. Nach Meinung der Befragten darf das Fundingziel einer Kampagne im Mittel um 32,6 \% höher angesetzt werden als die tatsächlich benötigte Summe. 
Tab. 14 Einsatzbereitschaft signalisieren. (Quelle: Eigene Darstellung)

\begin{tabular}{l|l|l|l|l|l|l}
\hline $\begin{array}{l}\text { Signale der } \\
\text { Einsatzbereitschaft }\end{array}$ & 1. & 2. & 3. & 4. & 5. & 6. \\
\hline $\begin{array}{l}\text { Qualitativ hochwertiges } \\
\text { Pitch-Video }\end{array}$ & $11,11 \%$ & $9,52 \%$ & $7,94 \%$ & $14,29 \%$ & $29,98 \%$ & $30,16 \%$ \\
\hline $\begin{array}{l}\text { Direkte Kommunikation } \\
\text { mit der Crowd }\end{array}$ & $33,33 \%$ & $20,63 \%$ & $19,05 \%$ & $12,70 \%$ & $11,11 \%$ & $3,17 \%$ \\
\hline $\begin{array}{l}\text { Schnelle Beantwortung } \\
\text { von Fragen }\end{array}$ & $3,17 \%$ & $30,16 \%$ & $28,57 \%$ & $11,11 \%$ & $15,87 \%$ & $11,11 \%$ \\
\hline $\begin{array}{l}\text { Veröffentlichung von } \\
\text { regelmäßigen Updates }\end{array}$ & $11,11 \%$ & $23,81 \%$ & $15,87 \%$ & $31,75 \%$ & $11,11 \%$ & $6,35 \%$ \\
\hline $\begin{array}{l}\text { Fehlerfreie } \\
\text { Projektbeschreibung }\end{array}$ & $1,59 \%$ & $7,94 \%$ & $9,52 \%$ & $9,52 \%$ & $25,40 \%$ & $46,03 \%$ \\
\hline $\begin{array}{l}\text { Authentische } \\
\text { Darstellung der Idee/des } \\
\text { Projekts }\end{array}$ & $39,68 \%$ & $7,94 \%$ & $19,05 \%$ & $20,63 \%$ & $9,52 \%$ & $3,17 \%$ \\
\hline
\end{tabular}

Ein stagnierender Zufluss von Unterstützungsbeiträgen könnte dazu führen, dass eine Kampagne an Attraktivität verliert. Als Mittel, um diesem Aspekt entgegenwirken zu können, wurden die Veröffentlichung von Updates (51\%), Angebot zusätzlicher Belohnungen (Goodies) (22\%) und der Veranstaltung eines Wettbewerbs (19\%) benannt (Abb. 6).

Abschließend wurden die Befragten gebeten, die entsprechenden projektspezifischen Erfolgsfaktoren der Projektgestaltung hinsichtlich ihrer Wichtigkeit zu ordnen (Anhang Tab. 16). Hierbei wurde als zentraler Erfolgsfaktor die innovative Projektidee genannt $(58,1 \%)$. Somit ist eine innovative Projektidee ein Kernfaktor, um im $\mathrm{CF}$ erfolgreich sein zu können.

\section{$6 \quad$ Zusammenfassung}

Auf Basis der vorliegenden Untersuchung wurden unterschiedliche Einflussfaktoren auf den Erfolg einer Kampagne - das Erreichen des Finanzierungsziels untersucht. Im Kern lag die Untersuchung dabei auf der qualitativen Einschätzung von Experten der vier zentralen Interessengruppen des CF. Die Ergebnisse spiegeln zum Teil die Ergebnisse der Literatur wider. Durch den vorliegenden Ansatz konnten weitere Ansichten mit tiefergehenden Argumentationen betrachtet werden.

Im Grundsatz zeigt sich, dass eine innovative Idee der Ausgangspunkt des weiteren Handelns sein sollte. Ist ein gewisser Innovationsgrad nicht vorhanden, 


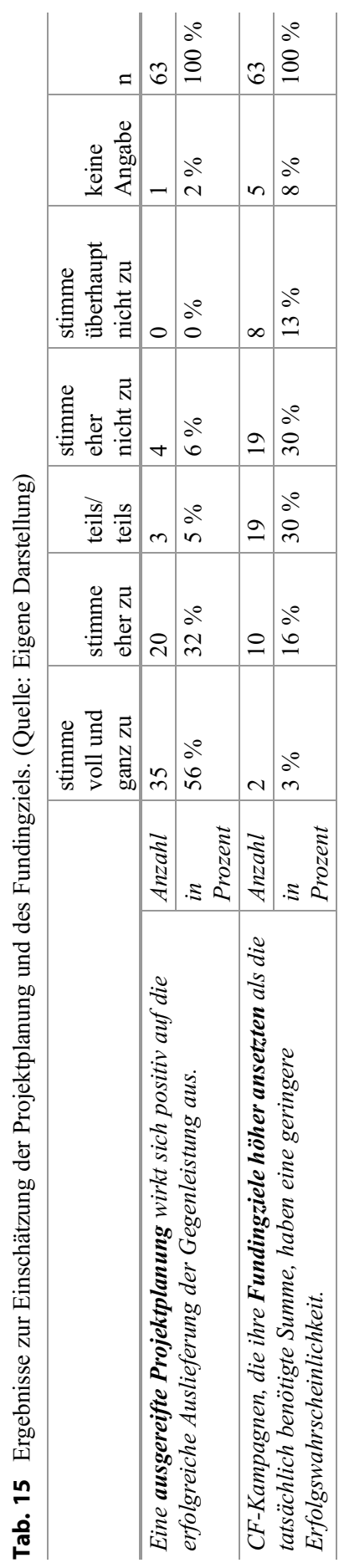




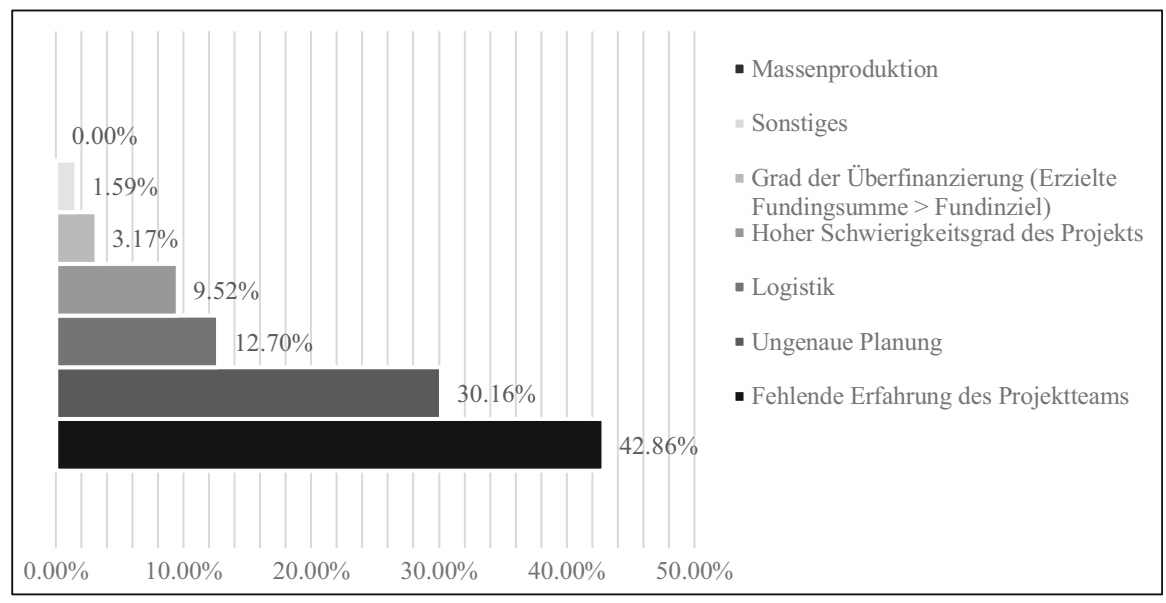

Abb. 5 Verspätete Auslieferung der Gegenleistung. (Quelle: Eigene Darstellung)

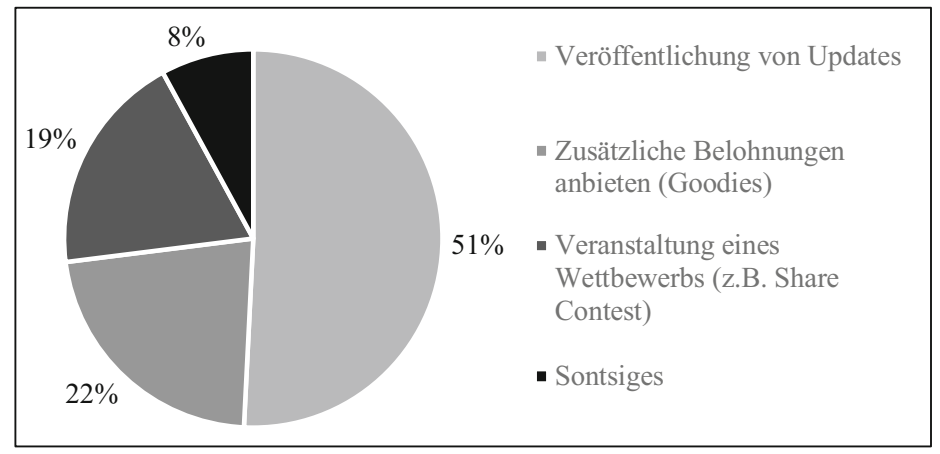

Abb. 6 Attraktivität der Kampagne. (Quelle: Eigene Darstellung)

so kann auch bei großer Bemühung und Einhaltung aller zentralen Erfolgsfaktoren, eine Kampagne scheitern.

Darüberhinaus spielt z. B. die Größe des sozialen Netzwerks eine bedeutende Rolle im CF. Während jedoch die Literatur und Plattform-Empfehlungen darauf zielen, dass Initiatoren ihre privaten Social Media Profile verknüpfen, zeigen unsere Ergebnisse auf, dass Initiatoren für ihre Kampagne eine eigene öffentliche Facebook-Fanseite verknüpfen sollten. Dieses Medium stellt zudem eine kostengünstige bzw. kostenlose Kommunikationsplattform zur Verfügung, um u. a. auch eine Community zur Kampagne aufbauen und eine aktive Kommunikation betreiben 
zu können. Vor allem letzteres wird als Schlüsselfaktor zur erfolgreichen Kampagne gesehen. Eng damit verknüpft ist auch das Vorhandensein eines Projektteams. Zentral an diesem Gedanken ist jedoch nicht, dass eine auffallende Arbeitslast von mehreren Personen aufgefangen werden kann, sondern vielmehr der Gedanke, dass ein größeres soziales Netzwerk bereits unmittelbar zum Kampagnenstart vorhanden ist.

Die Qualifikation des Initiators/Projektteams spielt im equity-based CF eine große Rolle, da hierbei gewisse wirtschaftliche Interessen verfolgt werden, die betriebswirtschaftliche Kenntnisse voraussetzen. Für das reward-based CF konnte diese Bedeutung nicht ermittelt werden. Dies begründet sich u. a. darin, dass bei dieser Form des CF oftmals die Idee im Vordergrund steht und solche Projekte häufig nicht als nachhaltige Geschäftsmodelle gesehen werden.

Bei der Nutzung von Kanälen zur Promotion einer Kampagne stehen die sozialen Netzwerke und Mund-zu-Mund-Kommunikation bei der vorliegenden Expertenbefragung an erster Stelle. Empfehlungen aus der Literatur, auch Kanäle wie TV oder Radio zu nutzen, um mehr potenzielle Unterstützer zu erreichen, werden von den Befragten nur teilweise bestätigt. Dabei wird TV als eher ungeeignet angesehen. Zur Aufrechterhaltung der Attraktivität einer Kampagne sind regelmäßige Updates zielführend. Eine Empfehlung der Befragten lautet hier, im Mittel 6,3 Updates zu veröffentlichen. Bei einer Laufzeit von 30 Tagen würde dies bedeuten, dass alle 4-5 Tage ein Update veröffentlicht werden sollte. Mit solchen Updates können zudem Zuflüsse der Unterstützungsbeiträge bei einer Stagnation angekurbelt werden.

Eine gelungene Projektbeschreibung wirkt sich gemäß der Untersuchung positiv auf den Erfolg einer Kampagne aus. Hierbei spielen Attribute wie Begeisterung, Authentizität, Verständlichkeit und Transparenz eine tragende Rolle. Die Kreativität und die Fehlerfreiheit einer Projektbeschreibung stehen für die Befragten jedoch weniger im Fokus als in der Literatur und von Plattformen empfohlen wird. Eine Projektbeschreibung beinhaltet auch die visuelle Gestaltung einer Kampagne. Bilder und Grafiken sind gegenüber Texten zu bevorzugen. Zudem hilft ein gelungenes Pitch-Video die Erfolgschancen zu steigern. Für die Erstellung eines solchen Videos ist das Budget für die Produktion kein Hindernis. Viel wichtiger ist für die Befragten, die Aufklärung des Vorhabens und die authentische Darstellung der Initiatoren und des Produktes oder der Projektidee. Die Dauer von 3-4 Minuten sollte auch hier nicht überschritten werden.

Ein weiterer zentraler Bereich beschäftigt sich mit der Planung einer Kampagne. So ist z. B. das Fundingziel ein empfindlicher Faktor. Das richtige Maß zu finden ist schwierig. Das Fundingziel zu hoch anzusetzen, kann laut Literatur die Erfolgschancen schmälern. Gemäß der Befragung hingegen kann auch ein Erfolg eintreten, 
wenn das Fundingziel die tatsächlich benötige Summe übersteigt. Die Laufzeit einer Kampagne sollte entsprechend des Unterstützernetzwerks gewählt werden. Gegenüber US-Plattformen wie Kickstarter, die über eine Vielzahl an potenziellen Unterstützern verfügen, ist die Zahl der potenziellen Unterstützer auf Plattformen wie Startnext deutlich beschränkter. Dies spiegeln auch die Ergebnisse wider. Gegenüber einer Laufzeit bei Kickstarter - von ca. 30 Tagen - empfehlen die Befragten eine Laufzeit von rund 48 Tagen. Dies begründet sich u. a. darin, dass die Befragten grundsätzlich einer deutschen CF-Community angehören. Das Erreichen des angestrebten Finanzierungsbetrages dauert auf z. B. Startnext länger als auf Kickstarter, was der geringen Unterstützerzahl geschuldet ist.

Die aus der vorliegenden Expertenbefragung qualitativ ermittelten Forschungsergebnisse des reward-based CF spiegeln zum einen zu Teilen die Ergebnisse bestehender Forschung wider, ermöglichen aber zugleich, tiefergehende und qualitative Fragestellungen zu beatworten. Dieses Vorgehen schafft einen wertvollen Beitrag zur Theorie und Praxis und hilft somit die langfristige Nachhaltigkeit des rewardbased CF zu gewährleisten. Darüberhinaus werden durch diese Arbeit bestehende Forschungsergebnisse mit bestehenden theoriegeleiteten Ergebnissen abgeglichen. Dies ermöglicht das Abgleichen der Standhaftigkeit bestehender Erfolgsfaktoren in einem sich zunehmend weiterentwickelnden Umfeld, z. B. der Erfahrungssteigerung (positiv und negativ) von Projektunterstützern. Aus praktischer Sicht helfen diese Ergebnisse Projektinitiatoren dabei, ihre Kampagne mit einer höheren Erfolgswahrscheinlichkeit zu gestalten. Plattformanbieter können diese Ergebnisse aufgreifen und sie in Richtlinien für „Best Practices“ verarbeiten.

Die Limitation dieser Untersuchung können u. a. darin gesehen werden, dass im Wesentlichen nur Erfolgsfaktoren betrachtet wurden, die den Erfolg hinsichtlich des Erreichens des Finanzierungsziels definieren. Eine Betrachtung darüberhinaus wäre von Bedeutung, um die Ganzheitlichkeit dieses Feldes abdecken zu können. Zudem ist weiterhin fraglich, ob diese Ergebnisse auch auf andere Formen des CF repliziert werden und ob die ermittelten Ergebnisse auch in einer englischsprachigen Community repliziert werden können.

\section{Anhang A.1 Rangfolge der projektspezifischen Erfolgsfaktoren}




\begin{tabular}{|c|c|c|c|c|c|c|c|c|c|c|c|}
\hline ก่ & 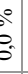 & $\begin{array}{l}\text { o } \\
\text { ñ. } \\
\text { s. }\end{array}$ & $\begin{array}{l}\text { oे } \\
\text { in } \\
\text { o. }\end{array}$ & $\begin{array}{l}0 \\
0 \\
0 \\
0\end{array}$ & o. & $\begin{array}{l}0 \\
\vdots \\
\stackrel{0}{-}\end{array}$ & \begin{tabular}{l}
0 \\
$\vdots$ \\
\hdashline
\end{tabular} & $\begin{array}{l}0 \\
0 \\
0 \\
-1\end{array}$ & $\begin{array}{l}\stackrel{0}{a} \\
\tilde{n}^{2}\end{array}$ & $\begin{array}{l}\stackrel{0}{ } \\
\text { in } \\
\tilde{n}^{2}\end{array}$ & $\begin{array}{l}\stackrel{0}{2} \\
\sim \\
\tilde{m}\end{array}$ \\
\hline 2 & 更 & $\begin{array}{l}0 \\
\stackrel{0}{\circ} \\
\infty \\
+\end{array}$ & $\begin{array}{l}0 \\
\text { in } \\
\text { ñ. }\end{array}$ & $\begin{array}{l}\stackrel{0}{2} \\
\tilde{N}^{2}\end{array}$ & $\begin{array}{l}a^{\circ} \\
n \\
b^{2}\end{array}$ & $\begin{array}{l}\stackrel{0}{\circ} \\
\stackrel{0}{-}\end{array}$ & $\begin{array}{l}\stackrel{0}{0} \\
\dot{0} \\
\stackrel{-}{0}\end{array}$ & $\left|\begin{array}{c}0 \\
\vdots \\
n \\
0 \\
0\end{array}\right|$ & $\begin{array}{l}\stackrel{\circ}{\circ} \\
\therefore \\
8\end{array}$ & $\begin{array}{l}0 \\
0 \\
0 \\
0 \\
-i\end{array}$ & $\begin{array}{l}\therefore \\
\stackrel{0}{c} \\
\tilde{m}\end{array}$ \\
\hline$\stackrel{\infty}{\infty}$ & $\stackrel{0}{\circ}$ & $\begin{array}{l}\stackrel{0}{\circ} \\
\mathrm{m}^{2}\end{array}$ & $\begin{array}{l}\circ \\
\stackrel{0}{0} \\
\stackrel{-}{-}\end{array}$ & $\begin{array}{l}0 \\
i n \\
n \\
0\end{array}$ & $\frac{\partial^{\circ}}{\infty^{\circ}}$ & $\begin{array}{l}0 \\
\vdots \\
\stackrel{0}{-}\end{array}$ & 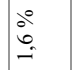 & $\begin{array}{l}0 \\
2 \\
2\end{array}$ & $\begin{array}{l}\stackrel{0}{\circ} \\
\infty \\
\infty \\
+\end{array}$ & $\begin{array}{l}\dot{0}^{\circ} \\
0 \\
0^{\circ}\end{array}$ & $\frac{0}{a}$ \\
\hline$\therefore$ & $\begin{array}{l}0 \\
\vdots \\
0 \\
0\end{array}$ & $\begin{array}{l}{ }_{0}^{\circ} \\
\therefore\end{array}$ & $\begin{array}{l}\circ \\
0^{\circ} \\
\infty \\
\dot{\sigma}^{+}\end{array}$ & $\begin{array}{l}0 \\
0 \\
0 \\
-1\end{array}$ & $\begin{array}{l}0 \\
\vdots \\
0 \\
-i\end{array}$ & $\begin{array}{l}\stackrel{0}{\circ} \\
\text { in }\end{array}$ & $\begin{array}{l}0 \\
\stackrel{0}{0} \\
-1\end{array}$ & $\frac{0}{\infty^{\circ}}$ & 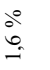 & $\begin{array}{l}a^{0} \\
n \\
0 \\
0\end{array}$ & $\begin{array}{l}\circ \\
a \\
a\end{array}$ \\
\hline$\stackrel{\circ}{0}$ & $\begin{array}{l}0 \\
: \\
0 \\
0\end{array}$ & 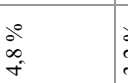 & $\stackrel{\circ}{\stackrel{0}{m}}$ & 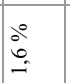 & $\begin{array}{l}\dot{0}^{\circ} \\
\infty \\
+\end{array}$ & $\begin{array}{l}\stackrel{0}{2} \\
\text { in } \\
\dot{I}\end{array}$ & \begin{tabular}{l}
$\stackrel{0}{0}$ \\
$\infty$ \\
\hdashline
\end{tabular} & $\begin{array}{c}0 \\
0 \\
0 \\
-1\end{array}$ & 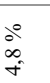 & $\begin{array}{l}{ }^{\circ} \\
\text { in } \\
0\end{array}$ & $\frac{\therefore}{\infty^{\circ}}$ \\
\hline$\because 2$ & $\begin{array}{l}0 \\
\vdots \\
0 \\
0\end{array}$ & $\begin{array}{l}\overbrace{0}^{\circ} \\
\text { o }\end{array}$ & $\begin{array}{l}\circ \\
0 \\
0\end{array}$ & \begin{tabular}{l}
$d^{\circ}$ \\
$b$ \\
\hdashline
\end{tabular} & $\frac{\partial^{\circ}}{\infty^{\circ}}$ & $\begin{array}{l}\stackrel{0}{2} \\
\mathrm{~m}^{2}\end{array}$ & $\begin{array}{l}\therefore 0 \\
\stackrel{0}{-}\end{array}$ & $\begin{array}{l}\therefore \\
\stackrel{0}{3} \\
=\end{array}$ & $\begin{array}{l}\stackrel{\circ}{0} \\
0_{0}^{\circ}\end{array}$ & $\begin{array}{l}\partial^{\circ} \\
\text { in } \\
0\end{array}$ & 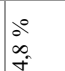 \\
\hline \pm 3 & $\begin{array}{l}a^{\circ} \\
m^{2}\end{array}$ & 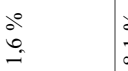 & $\frac{\therefore}{\infty}$ & $\begin{array}{l}0 \\
\stackrel{0}{0} \\
\stackrel{\sim}{\sim}\end{array}$ & $\begin{array}{l}\stackrel{0}{\circ} \\
\infty \\
\dot{f}\end{array}$ & $\frac{0^{\circ}}{\infty^{\circ}}$ & $\begin{array}{l}\stackrel{0}{2} \\
\stackrel{m}{m}\end{array}$ & $\frac{\partial^{0}}{\infty^{\circ}}$ & $\begin{array}{l}\stackrel{0}{2} \\
\stackrel{m}{=}\end{array}$ & $\begin{array}{l}\dot{0}^{\circ} \\
\infty \\
\dot{f}\end{array}$ & $\begin{array}{l}0 \\
\infty \\
\infty \\
+\end{array}$ \\
\hline$\stackrel{9}{9}$ & $\begin{array}{l}0 \\
\vdots \\
0 \\
0\end{array}$ & $\begin{array}{l}\stackrel{\circ}{\circ} \\
\stackrel{m}{m}\end{array}$ & do & $\begin{array}{l}\stackrel{0}{2} \\
\text { ì } \\
\text { min }\end{array}$ & $\begin{array}{l}\stackrel{0}{0} \\
\infty \\
+\end{array}$ & $\begin{array}{l}\stackrel{0}{0} \\
\infty \\
\dot{f}\end{array}$ & $\frac{\therefore}{\infty}$ & $\begin{array}{l}\circ \\
\therefore \\
\infty \\
\forall\end{array}$ & $\begin{array}{l}\stackrel{\circ}{\circ} \\
\text { ì } \\
\text { mे }\end{array}$ & $\begin{array}{l}\circ \\
\stackrel{0}{0} \\
\infty\end{array}$ & $\begin{array}{l}\therefore 0 \\
\text { ì }\end{array}$ \\
\hline I & $\begin{array}{l}0 \\
\vdots \\
0 \\
0\end{array}$ & $\begin{array}{l}\stackrel{0}{0} \\
\vdots \\
0\end{array}$ & $\begin{array}{l}\stackrel{\circ}{\circ} \\
\text { cे } \\
m^{2}\end{array}$ & $\begin{array}{l}0 \\
\therefore \\
0\end{array}$ & $\begin{array}{l}\circ \\
\dot{0}^{\circ} \\
\infty\end{array}$ & $\begin{array}{l}\dot{ }^{\circ} \\
\infty \\
\dot{\sigma}\end{array}$ & $\frac{\therefore}{\infty}$ & $\begin{array}{l}0 \\
0 \\
1 \\
0^{\circ}\end{array}$ & $\frac{\stackrel{\circ}{0}^{\circ}}{\infty^{\circ}}$ & $\frac{a}{a}$ & $\frac{\therefore}{\infty^{\circ}}$ \\
\hline$=-$ & $\begin{array}{l}0 \\
b \\
0 \\
-\end{array}$ & $\frac{\partial^{\circ}}{\infty}$ & $\begin{array}{l}\stackrel{\circ}{ } \\
\text { ì } \\
\text { m}\end{array}$ & 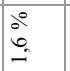 & $\begin{array}{l}\dot{ }^{\circ} \\
\infty \\
\dot{f}\end{array}$ & $\begin{array}{l}\stackrel{0}{0} \\
\infty \\
\dot{f}\end{array}$ & $\begin{array}{l}a^{\circ} \\
n \\
b^{2}\end{array}$ & $\frac{\partial^{\circ}}{\infty^{\circ}}$ & 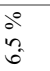 & $\begin{array}{l}0 \\
\dot{0}^{\circ} \\
\infty \\
\dot{f}\end{array}$ & $\begin{array}{l}0 \\
\infty \\
\infty \\
+\end{array}$ \\
\hline$\stackrel{\ominus}{9}$ & $\begin{array}{c}0 \\
b_{0}^{\circ} \\
-i\end{array}$ & 宂 & $\begin{array}{l}\text { å } \\
\text { in } \\
b^{2}\end{array}$ & $\frac{\circ}{\infty^{\circ}}$ & $\frac{a}{2}$ & $\begin{array}{l}0 \\
\infty \\
\infty \\
\dot{f}\end{array}$ & $\begin{array}{l}\stackrel{\circ}{2} \\
\sim \\
m\end{array}$ & $\begin{array}{l}\stackrel{0}{2} \\
\stackrel{m}{m}\end{array}$ & $\begin{array}{l}\text { 足 } \\
\text { in } \\
\text { - }\end{array}$ & $\begin{array}{l}0 \\
\text { in } \\
i \\
6\end{array}$ & $\begin{array}{l}\stackrel{\circ}{\circ} \\
\text { iे } \\
m^{2}\end{array}$ \\
\hline$\sigma^{\circ}$ & $\begin{array}{c}0 \\
0 \\
-1\end{array}$ & $\begin{array}{l}\stackrel{0}{\circ} \\
\infty \\
+\end{array}$ & 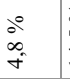 & $\begin{array}{l}\text { वे } \\
\text { in } \\
\text { f }\end{array}$ & $\begin{array}{l}\stackrel{0}{ } \\
\infty \\
+\end{array}$ & $\begin{array}{l}\partial^{\circ} \\
\text { in }\end{array}$ & $\stackrel{\circ}{0}$ & $\begin{array}{l}\stackrel{0}{2} \\
\dot{m} \\
m\end{array} \mid$ & $\frac{\partial^{\circ}}{\infty^{\circ}}$ & $\begin{array}{l}\text { 足 } \\
\text { in }\end{array}$ & $\begin{array}{l}\stackrel{0}{2} \\
\stackrel{m}{m}\end{array}$ \\
\hline$\infty$ & $\begin{array}{l}\stackrel{0}{\circ} \\
m^{2}\end{array}$ & $\begin{array}{l}\text { 足 } \\
\text { in } \\
\text { o }\end{array}$ & $\frac{\partial^{\circ}}{\infty}$ & $\begin{array}{l}0 \\
\stackrel{0}{2} \\
\tilde{m}^{2}\end{array}$ & $\begin{array}{l}0 \\
0 \\
i \\
0\end{array}$ & $\begin{array}{l}\stackrel{0}{ } \\
\text { ì }\end{array}$ & $\begin{array}{l}0 \\
\stackrel{0}{0} \\
-\end{array}$ & $\mid \begin{array}{c}0 \\
2 \\
n \\
0\end{array}$ & $\begin{array}{l}\stackrel{\circ}{\circ} \\
\text { mे }\end{array}$ & $\begin{array}{l}0 \\
\vdots \\
0 \\
-1\end{array}$ & $\begin{array}{l}0 \\
\infty \\
\infty \\
\infty \\
+\end{array}$ \\
\hline$\therefore$ & $\begin{array}{l}0^{\circ} \\
m^{2}\end{array}$ & $\begin{array}{l}\text { o̊ } \\
\text { in- }\end{array}$ & $\begin{array}{l}\stackrel{2}{\circ} \\
\mathrm{a}^{\circ}\end{array}$ & \begin{tabular}{l}
0 \\
0 \\
0 \\
\hdashline
\end{tabular} & $\frac{\therefore}{\infty}$ & $\begin{array}{l}\stackrel{\circ}{2} \\
\text { ले }\end{array}$ & $\begin{array}{l}\stackrel{0}{0} \\
\stackrel{0}{-}\end{array}$ & $\mid \begin{array}{c}0 \\
\infty^{\circ}\end{array}$ & $\begin{array}{l}\text { 足 } \\
\text { in } \\
b^{2}\end{array}$ & $\begin{array}{l}\stackrel{0}{2} \\
\stackrel{m}{=}\end{array}$ & 家 \\
\hline 6 & $\begin{array}{l}0 \\
\mathrm{~m}^{\circ}\end{array}$ & $\begin{array}{l}{ }^{\circ} \\
\infty \\
+ \\
+\end{array}$ & $\begin{array}{l}0 \\
\stackrel{0}{ } \\
\infty \\
+\end{array}$ & $\begin{array}{l}\stackrel{0}{2} \\
\tilde{n}^{2}\end{array}$ & $\frac{\partial^{\circ}}{\infty^{\circ}}$ & $\begin{array}{l}a^{\circ} \\
n \\
b^{2}\end{array}$ & $\begin{array}{l}\stackrel{0}{0} \\
\infty \\
\sim\end{array}$ & $\begin{array}{l}0 \\
0 \\
0^{\circ}\end{array}$ & $\begin{array}{l}\frac{\circ}{2} \\
a^{\circ}\end{array}$ & $\begin{array}{l}\stackrel{0}{ } \\
i \\
m\end{array}$ & $\begin{array}{l}0 \\
\infty \\
\infty \\
+\end{array}$ \\
\hline$i$ & $\begin{array}{l}0_{0}^{\circ} \\
\dot{0} \\
0^{\circ}\end{array}$ & $\begin{array}{l}\stackrel{\circ}{\circ} \\
\infty \\
\dot{\sigma}\end{array}$ & $\begin{array}{l}{ }^{0} \\
\vdots \\
0\end{array}$ & $\begin{array}{l}\stackrel{0}{2} \\
\text { in } \\
m^{2}\end{array}$ & $\begin{array}{l}\therefore \\
\text { ju } \\
m\end{array}$ & $\begin{array}{l}\stackrel{0}{a} \\
2\end{array}$ & $\begin{array}{l}\stackrel{0}{1} \\
\text { İ }\end{array}$ & $\begin{array}{c}0 \\
0 \\
\infty \\
\dot{\sigma}\end{array}$ & $\frac{\circ}{\infty^{\circ}}$ & $\begin{array}{l}\dot{0}^{\circ} \\
\infty \\
+\end{array}$ & $\frac{\therefore}{\infty}$ \\
\hline$\dot{+}$ & $\begin{array}{c}0 \\
0 \\
\text { iे } \\
m^{2}\end{array}$ & $\begin{array}{l}\text { o } \\
\text { in } \\
\text { s. }\end{array}$ & $\begin{array}{l}\stackrel{0}{\circ} \\
\stackrel{2}{=}\end{array}$ & $\frac{\partial^{\circ}}{\infty^{\circ}}$ & $\begin{array}{l}\stackrel{0}{0} \\
\infty \\
\dot{\sigma}\end{array}$ & $\begin{array}{l}\stackrel{0}{0} \\
\infty \\
+\end{array}$ & $\begin{array}{l}\grave{0} \\
\text { mे }\end{array}$ & $\left|\begin{array}{c}0 \\
0 \\
0 \\
\dot{f}\end{array}\right|$ & $\begin{array}{l}{ }^{\circ} \\
\text { in } \\
b^{2}\end{array}$ & $\frac{\partial 0}{\infty^{0}}$ & 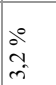 \\
\hline$\dot{m}$ & $\begin{array}{l}\mathrm{c} \\
\mathrm{m}^{2}\end{array}$ & $\frac{\circ}{a}$ & $\begin{array}{l}\overbrace{0}^{\circ} \\
\stackrel{0}{\circ}\end{array}$ & $\frac{\circ}{\circ}$ & $\begin{array}{l}\stackrel{0}{2} \\
\text { mj }\end{array}$ & $\begin{array}{l}\dot{0}^{\circ} \\
\text { in }\end{array}$ & $\begin{array}{l}\stackrel{0}{0} \\
\infty \\
\dot{\sigma}\end{array}$ & $\begin{array}{l}0 \\
0 \\
0 \\
-1\end{array}$ & $\begin{array}{l}0^{\circ} \\
\text { o } \\
+\end{array}$ & $\frac{\partial^{\circ}}{\infty^{\circ}}$ & $\stackrel{0}{0}_{0}^{\circ}$ \\
\hline i & $\therefore$ & $\therefore$ & $\begin{array}{l}\stackrel{0}{\circ} \\
\vdots \\
\sigma^{\circ}\end{array}$ & $\begin{array}{l}\stackrel{0}{ } \\
\text { İ } \\
\text { I }\end{array}$ & 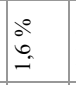 & $\begin{array}{l}\stackrel{0}{ } \\
\infty \\
+\end{array}$ & $\begin{array}{l}\stackrel{0}{\circ} \\
\cong\end{array}$ & $\begin{array}{l}\therefore \\
\therefore \\
0^{\circ}\end{array}$ & $\begin{array}{l}\therefore \\
\therefore \\
0\end{array}$ & $\begin{array}{l}\stackrel{0}{0} \\
\stackrel{0}{-}\end{array}$ & $\begin{array}{l}\stackrel{0}{\circ} \\
\vdots \\
0\end{array}$ \\
\hline$-i s$ & $\circ$ & $\frac{\stackrel{\circ}{\infty}}{\infty}$ & $\begin{array}{l}\stackrel{2}{\circ} \\
\text { ले }\end{array}$ & $\frac{\circ}{\infty^{\circ}}$ & $\begin{array}{l}\therefore \\
\therefore \\
0\end{array}$ & 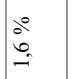 & $\frac{\therefore}{\infty}$ & $\begin{array}{c}\stackrel{0}{0} \\
\dot{0} \\
-1\end{array}$ & $\begin{array}{l}\stackrel{\circ}{ } \\
\text { ì }\end{array}$ & $\begin{array}{l}\dot{0} \\
\dot{0} \\
0\end{array}$ & $\begin{array}{l}\therefore \\
\vdots \\
0\end{array}$ \\
\hline 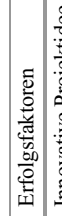 & & 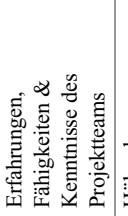 & 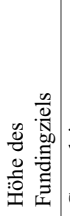 & 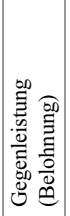 & 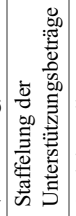 & 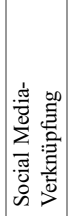 & 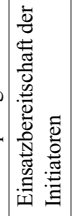 & 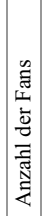 & 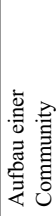 & 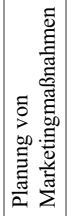 & 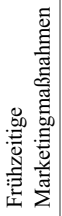 \\
\hline
\end{tabular}




\begin{tabular}{|c|c|c|c|c|c|c|c|c|}
\hline$\partial^{\circ}$ & $\begin{array}{l}\stackrel{0}{2} \\
\text { ले }\end{array}$ & $\begin{array}{l}\circ \\
0 \\
0\end{array}$ & $\mid \begin{array}{l}0 \\
0 \\
0 \\
0\end{array}$ & & $\begin{array}{l}\stackrel{\circ}{\circ} \\
\vec{m}\end{array}$ & $\begin{array}{l}0 \\
0 \\
0\end{array}$ & $\begin{array}{l}\stackrel{0}{2} \\
\vdots \\
a\end{array}$ & $\frac{\partial^{\circ}}{\infty^{\circ}}$ \\
\hline$\Rightarrow$ & $\begin{array}{l}\partial^{\circ} \\
n^{2}\end{array}$ & $\begin{array}{l}\stackrel{\circ}{2} \\
\text { ì }\end{array}$ & $\begin{array}{c}0 \\
0 \\
0 \\
c\end{array}$ & $\begin{array}{l}\stackrel{\circ}{\circ} \\
\text { mे } \\
m^{2}\end{array}$ & \begin{tabular}{l} 
¿ \\
in \\
\multirow{\Xi}{*}{}
\end{tabular} & $\begin{array}{l}\dot{0}^{\circ} \\
\infty \\
\dot{f}\end{array}$ & $\begin{array}{l}\AA^{\circ} \\
i_{0}^{2}\end{array}$ & $\frac{a}{a}$ \\
\hline $\begin{array}{l}0^{\circ} \\
\infty \\
\forall \\
+\end{array}$ & $\begin{array}{l}\dot{ }^{\circ} \\
\infty \\
+\end{array}$ & $\begin{array}{l}\stackrel{\circ}{\circ} \\
\dot{\sigma} \\
\dot{\sigma}\end{array}$ & $\begin{array}{l}\dot{0} \\
\dot{0} \\
\dot{\tau}\end{array}$ & $\begin{array}{l}0 \\
\therefore \\
0 \\
0\end{array}$ & $\begin{array}{l}\stackrel{0}{\circ} \\
\text { בे }\end{array}$ & $\begin{array}{l}0 \\
\stackrel{0}{0} \\
\stackrel{-}{-}\end{array}$ & $\begin{array}{l}\stackrel{0}{ } \\
\grave{I}\end{array}$ & $\frac{\partial^{\circ}}{\infty^{\circ}}$ \\
\hline $\begin{array}{l}{ }^{\circ} \\
\text { à }\end{array}$ & $\begin{array}{l}{ }^{\circ} \\
\text { in. } \\
\text { b. }\end{array}$ & $\begin{array}{l}\stackrel{0}{\circ} \\
\dot{m} \\
\dot{m}\end{array}$ & $\begin{array}{l}0 \\
2 \\
n \\
0\end{array}$ & $\begin{array}{l}\therefore \\
\vdots \\
a\end{array}$ & & $\begin{array}{l}\stackrel{0}{2} \\
\mathrm{~N}^{2}\end{array}$ & $\begin{array}{l}\stackrel{0}{0} \\
\text { in } \\
0\end{array}$ & $\begin{array}{l}0^{\circ} \\
\text { in } \\
b^{2}\end{array}$ \\
\hline $\begin{array}{l}\stackrel{\circ}{\circ} \\
a^{\circ}\end{array}$ & $\begin{array}{l}0 \\
0 \\
0 \\
-\end{array}$ & $\frac{\circ}{2}$ & $\begin{array}{l}2 \\
\text { in } \\
\text { m. }\end{array}$ & 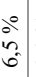 & $\begin{array}{l}\stackrel{8}{\circ} \\
\therefore\end{array}$ & \begin{tabular}{c}
0 \\
0 \\
0 \\
\hdashline
\end{tabular} & $\frac{\therefore}{\infty^{\circ}}$ & $\begin{array}{l}\stackrel{0}{0} \\
\infty \\
\dot{\theta}\end{array}$ \\
\hline $\begin{array}{l}a^{\circ} \\
\infty \\
\forall \\
\forall\end{array}$ & $\begin{array}{l}\stackrel{0}{ } \\
\text { בे }\end{array}$ & $\begin{array}{l}\stackrel{0}{2} \\
i^{2}\end{array}$ & $\begin{array}{l}0 \\
\infty \\
\infty \\
+\end{array}$ & $\begin{array}{c}\therefore^{\circ} \\
\frac{\infty^{\circ}}{}\end{array}$ & & $\frac{\partial^{\circ}}{\infty^{\circ}}$ & $\begin{array}{l}\therefore 0 \\
\therefore \\
a\end{array}$ & $\begin{array}{l}0 \\
0 \\
0 \\
0\end{array}$ \\
\hline 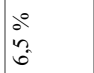 & $\begin{array}{l}0 \\
\dot{0}^{\circ} \\
\infty \\
\dot{\sigma}\end{array}$ & $\stackrel{\circ}{\circ} \underset{0}{0}$ & $\begin{array}{l}0 \\
\dot{2} \\
\tilde{m}^{2}\end{array}$ & $\begin{array}{c}\circ \\
\stackrel{0}{\circ} \\
m^{2}\end{array}$ & $\begin{array}{l}\text { 足 } \\
\text { in } \\
b^{2}\end{array}$ & & $\begin{array}{l}\text { वे } \\
\text { in } \\
6\end{array}$ & $\begin{array}{l}0 \\
0 \\
0 \\
-1\end{array}$ \\
\hline $\begin{array}{l}\text { 足 } \\
\text { in }\end{array}$ & $\begin{array}{l}\stackrel{\circ}{\circ} \\
\vdots \\
\dot{f}\end{array}$ & $\begin{array}{l}\stackrel{\circ}{\circ} \\
\stackrel{m}{m}\end{array}$ & $\begin{array}{l}\circ \\
\stackrel{0}{=} \\
=\end{array}$ & $\frac{\partial^{\circ}}{\infty^{\circ}}$ & $\begin{array}{l}0 \\
\text { o. } \\
\text { - }\end{array}$ & $\begin{array}{l}\stackrel{0}{0} \\
\infty \\
+\end{array}$ & $\begin{array}{l}\stackrel{0}{0} \\
\infty \\
f\end{array}$ & $\begin{array}{l}\stackrel{0}{\circ} \\
\stackrel{m}{m}\end{array}$ \\
\hline 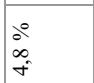 & $\begin{array}{l}\stackrel{0}{2} \\
\mathrm{~m}^{2}\end{array}$ & $\begin{array}{l}\stackrel{0}{0} \\
\stackrel{3}{\Rightarrow}\end{array}$ & 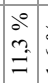 & 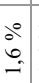 & $\begin{array}{l}\overbrace{0}^{\circ} \\
\text { o. }\end{array}$ & $\begin{array}{l}\stackrel{0}{2} \\
\mathrm{~m}^{2}\end{array}$ & $\begin{array}{l}\dot{0}^{\circ} \\
\infty \\
\dot{f}\end{array}$ & $\begin{array}{l}\therefore 0 \\
\infty \\
0 \\
0\end{array}$ \\
\hline$\frac{\therefore}{\infty^{\circ}}$ & $\frac{0^{\circ}}{\infty^{\circ}}$ & $\begin{array}{l}0 \\
i^{\circ} \\
n \\
0\end{array}$ & $\begin{array}{l}0 \\
\mathrm{~N}^{2} \\
\mathrm{~m}^{2}\end{array}$ & $\begin{array}{ll}0 \\
\stackrel{0}{0} \\
\stackrel{-}{-}\end{array}$ & 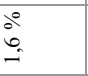 & $\begin{array}{l}\therefore \\
2 \\
a\end{array}$ & $\begin{array}{l}\stackrel{0}{ } \\
\mathrm{~m}^{2}\end{array}$ & $\begin{array}{l}\therefore \\
\stackrel{0}{0} \\
m\end{array}$ \\
\hline $\begin{array}{l}0 \\
\text { bo } \\
-1\end{array}$ & $\begin{array}{l}\stackrel{\circ}{\circ} \\
\infty \\
\dot{+}\end{array}$ & $\begin{array}{l}\dot{ }^{\circ} \\
\infty \\
\dot{\sigma}\end{array}$ & $\begin{array}{l}\therefore \\
\vdots \\
\grave{\jmath}\end{array}$ & 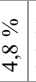 & $\begin{array}{l}\stackrel{\circ}{\circ} \\
\text { ले }\end{array}$ & $\begin{array}{l}\therefore \\
\therefore \\
0\end{array}$ & $\frac{2}{\infty^{\circ}}$ & $\begin{array}{l}\stackrel{0}{0} \\
\infty \\
\dot{\sigma}\end{array}$ \\
\hline 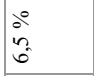 & $\begin{array}{l}a^{\circ} \\
\text { n. } \\
\delta^{2}\end{array}$ & $\begin{array}{l}\stackrel{\circ}{\circ} \\
\text { ì }\end{array}$ & $\frac{\partial^{\circ}}{\infty^{\circ}}$ & 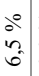 & $\begin{array}{l}{ }_{0}^{\circ} \\
\vdots \\
0\end{array}$ & $\begin{array}{l}\stackrel{0}{2} \\
\tilde{m}^{2}\end{array}$ & 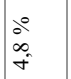 & $\begin{array}{l}0 \\
0 \\
0 \\
-1\end{array}$ \\
\hline $\begin{array}{l}0^{\circ} \\
\text { in } \\
b^{2}\end{array}$ & $\stackrel{0}{0}_{0}^{\circ}$ & $\frac{{ }^{\circ}}{\infty^{\circ}}$ & $\frac{\circ}{\infty^{\circ}}$ & $\begin{array}{l}\stackrel{0}{0} \\
\Rightarrow \\
\Rightarrow\end{array}$ & $\begin{array}{l}\therefore \\
\therefore \\
\therefore\end{array}$ & $\begin{array}{l}\stackrel{0}{2} \\
a^{\circ}\end{array}$ & $\begin{array}{l}\stackrel{0}{0} \\
\vdots \\
-1\end{array}$ & $\begin{array}{l}\stackrel{\circ}{0} \\
\infty \\
\dot{\sigma}\end{array}$ \\
\hline $\begin{array}{l}a^{\circ} \\
\text { o }\end{array}$ & $\begin{array}{l}\stackrel{\circ}{2} \\
\text { in } \\
\text { m. }\end{array}$ & $\frac{\circ}{\infty^{\circ}}$ & $\begin{array}{l}0 \\
0 \\
\infty \\
+\end{array}$ & 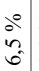 & $\begin{array}{l}\stackrel{8}{\circ} \\
\vdots \\
0\end{array}$ & $\begin{array}{l}\circ \\
\infty \\
\infty \\
+\end{array}$ & $\begin{array}{l}\therefore \\
i \\
\text { in }\end{array}$ & $\begin{array}{l}0 \\
i n \\
\text { in }\end{array}$ \\
\hline 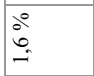 & $\begin{array}{l}\circ \\
\infty \\
\infty \\
+\end{array}$ & $\begin{array}{l}0 \\
i^{\circ} \\
n \\
0\end{array}$ & $\begin{array}{l}0 \\
\infty \\
\infty \\
+\end{array}$ & $\begin{array}{l}\therefore^{\circ} \\
\infty^{\prime}\end{array}$ & & & $\begin{array}{l}\therefore \\
i^{\circ} \\
m^{2}\end{array}$ & $\frac{\therefore}{\infty}$ \\
\hline $\begin{array}{l}0 \\
\stackrel{0}{\circ} \\
\infty \\
+\end{array}$ & $\begin{array}{l}\stackrel{\circ}{ } \\
\text { cे }\end{array}$ & $\begin{array}{l}\stackrel{0}{2} \\
\tilde{m}^{2}\end{array}$ & $\begin{array}{c}\circ \\
\dot{2} \\
\mathrm{~m}^{2}\end{array}$ & $\begin{array}{c}0 \\
\stackrel{0}{\circ} \\
\infty \\
+ \\
+\end{array}$ & 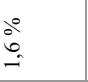 & $\frac{0^{\circ}}{\infty^{\circ}}$ & $\begin{array}{l}0 \\
\infty \\
\infty \\
\dot{f}\end{array}$ & $\begin{array}{l}\stackrel{0}{2} \\
\infty \\
\dot{\sigma}\end{array}$ \\
\hline $\begin{array}{l}\circ \\
\stackrel{\circ}{\circ} \\
-\end{array}$ & $\frac{\circ}{\infty}$ & $\frac{\circ}{\infty}$ & $\mid$ & $\begin{array}{c}\stackrel{0}{\circ} \\
\mathrm{m}\end{array}$ & 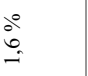 & $\begin{array}{l}\stackrel{0}{0} \\
\text { in } \\
m^{2}\end{array}$ & $\begin{array}{l}0 \\
\stackrel{0}{0} \\
\stackrel{-}{-1}\end{array}$ & $\begin{array}{l}0 \\
\infty \\
\infty \\
\dot{\sigma}\end{array}$ \\
\hline : & $\begin{array}{l}\stackrel{\circ}{\circ} \\
\text { ले } \\
\text { ले }\end{array}$ & $\frac{\circ}{\infty}$ & $\begin{array}{l}0 \\
\dot{0} \\
\mathrm{~m}^{2}\end{array}$ & 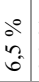 & $\begin{array}{l}\therefore \\
\therefore\end{array}$ & $\frac{\partial^{\circ}}{\infty^{\circ}}$ & $\begin{array}{l}\therefore \\
0 \\
0\end{array}$ & $\frac{0^{\circ}}{\infty^{\circ}}$ \\
\hline $\begin{array}{l}{ }^{\circ} \\
0_{0}^{\circ}\end{array}$ & $\frac{\partial^{\circ}}{\infty^{\circ}}$ & $\begin{array}{l}\stackrel{0}{2} \\
\stackrel{m}{m}\end{array}$ & $\begin{array}{l}0 \\
0 \\
n^{2}\end{array}$ & $\begin{array}{c}\circ \\
\stackrel{0}{\circ} \\
-\end{array}$ & $\stackrel{o}{0}_{0}^{\circ}$ & $\frac{\partial^{\circ}}{\infty^{\circ}}$ & $\begin{array}{l}0 \\
\therefore \\
0\end{array}$ & $\begin{array}{l}0 \\
0 \\
0\end{array}$ \\
\hline$\ddot{a}_{0}^{\circ}$ & $\begin{array}{l}\stackrel{0}{0}^{\circ} \\
0_{0}\end{array}$ & $\begin{array}{l}0 \\
0 \\
0 \\
0\end{array}$ & $\begin{array}{l}0 \\
0 \\
0 \\
0^{\prime}\end{array}$ & $\begin{array}{l}\stackrel{\circ}{\circ} \\
\vdots \\
0^{\circ}\end{array}$ & 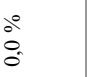 & $\begin{array}{l}0 \\
\infty \\
\infty \\
\dot{f}\end{array}$ & $\begin{array}{l}0 \\
\vdots \\
0 \\
0\end{array}$ & $\begin{array}{l}\stackrel{0}{2} \\
\stackrel{m}{m}\end{array}$ \\
\hline 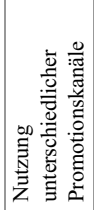 & 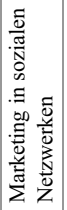 & 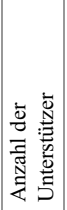 & 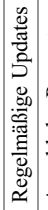 & 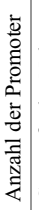 & 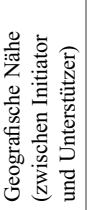 & 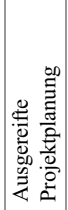 & 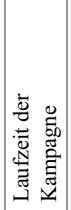 & 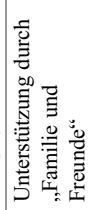 \\
\hline
\end{tabular}




\section{Literatur}

Agrawal, Ajay, Christian Catalini, und Avi Goldfarb. 2013. Some simple economics of crowdfunding. Cambridge, MA: National Bureau of Economic Research.

Ahlers, Gerrit K. C., Douglas Cumming, Christina Günther, und Denis Schweizer. 2015. Signaling in equity crowdfunding. Entrepreneurship: Theory and Practice 39(4): 955-980.

Backes-Gellner, Uschi, und Arndt Werner. 2007. Entrepreneurial signaling via education: A success factor in innovative start-ups. Small Business Economics 29(1-2): 173-190.

Belleflamme, Paul, Thomas Lambert, und Armin Schwienbacher. 2013a. Individual crowdfunding practices. Venture Capital 15(4): 313-333.

Belleflamme, Paul, Thomas Lambert, und Armin Schwienbacher. 2013b. Crowdfunding: Tapping the right crowd. Journal of Business Venturing 29(5): 585-609.

Blohm, Ivo, Eva Sieber, Moritz Schulz, Philipp Haas, Jan Marco Leimeister, und Karsten Wenzlaff. 2015. Crowdfunding 2020. Komplement oder Substitut für die Finanzindustrie? Universität St. Gallen, Institut für Wirtschaftsinformatik. Norderstedt: BoD - Books on Demand.

Cuhls, Kersten, Sybille Breiner, und Hariolf Grupp. 1995. Delphi-Bericht 1995 zur Entwicklung von Wissenschaft Technik - Mini Delphi. Fraunhofer Institut für Systemtechnik und Innovationsforschung.

Dalkey, Norman, und Olaf Helmer. 1963. An experimental application of the DELPHI method to the use of experts. Management Science 9(3): 458-467.

Dragonetti, Silvano, und Engelbert Weiss. 2015. Crowdfunding als neue Art der Finanzierung. In Unternehmensentwicklung. Strategien und Instrumente aus Forschung und Praxis, Hrsg. Philip Berchtold, Jochen Schellinger und Kim Oliver Tokarski. Bern: Springer Gabler.

Duffield, Christine. 1993. The Delphi technique: a comparison of results obtained using two expert panels. International Journal of Nursing Studies 30(3): 227-237.

Fueglistaller, Urs, Christoph Müller, und Thierry Volery, Hrsg. 2012. Gründungsfinanzierung. In Entrepreneurship, Wiesbaden: Springer Gabler.

Gafni, Hadar, Dan Marom, und Orly Sade. 2018. Are the life and death of a young start-up indeed in the power of the tongue? Lessons from online crowdfunding pitches. Strategic Entrepreneurship Journal 13(1): 3-23.

Gerber, Elizabeth, Julie Hui, und Pei-Yi Kuo. 2012. Crowdfunding: Why people are motivated to post and fund projects on crowdfunding platforms. In Proceedings of the international workshop on design, influence, and social technologies: Techniques, impacts and ethics. New York: ACM.

Häder, Michael. 2014. Delphi-Befragungen. Wiesbaden: Springer Fachmedien Wiesbaden.

Harzer, Alexandra. 2013. Erfolgsfaktoren im Crowdfunding. Ilmenau: Universitätsverlag Ilmenau (Menschen - Märkte - Medien - Management, 7).

Hong, Weiyin, James Y. L. Thong, und Kar Yan Tam. 2004. Designing product listing pages on e-commerce websites: An examination of presentation mode and information format. International Journal of Human-Computer Studies 61(4): 481-503.

Kollmann, Tobias. 2005. Finanzierung von jungen Unternehmen in der Net Economy. In Entrepreneurial Finance: Kompendium der Gründungs- und Wachstumsfinanzierung, Hrsg. Christoph J. Börne und Dietmar Grichnik. Heidelberg: Physica.

Kuppuswamy, Venkat, und Barry L. Bayus. 2018. Crowdfunding creative ideas: The dynamics of project backers in kickstarter. In The economics of crowdfunding, Hrsg. Douglas Cumming und Lars Hornuf. Wiesbaden: Springer Fachmedien Wiesbaden.

Kunz, Michael Marcin, Ulrich Bretschneider, Max Erler, und Jan Marco Leimeister. 2017: An empirical investigation of signaling in reward-based crowdfunding. Electronic Commerce Research 17(3): 425-461.

Lim, Eric T.K., Chee-Wee Tan, Dongback Seo, Dianne Cyr, und de Karsten Vries. 2013. An empirical investigation of the impact of online product presentation on hedonic web shopping. In The 12th annual pre-ICIS workshop on HCI research in MIS.

Lin, Mingfeng, und Siva Viswanathan. 2016. Home bias in online investments: An empirical study of an online crowdfunding market. Management science (im Druck). 
Lu, Chun-Ta, Sihong Xie, Xiangnan Kong, und Philip S Yu. 2014. Inferring the impacts of social media on crowdfunding. In The 7th ACM international conference, Hrsg. Carterette, Ben, Fernando Diaz, Carlos Castillo und Donald Metzler. New York.

Mollick, Ethan. 2014. The dynamics of crowdfunding: An exploratory study. Journal of Business Venturing 29(1): 1-16.

Mollick, Ethan, und Kuppuswamy Venkat. 2014. After the campaign: Outcomes of crowdfunding. UNC Kenan-Flagler Research Paper No.2376997. http://doi.org/10.2139/ssrn.2376997. Zugegriffen am 19.08.2016.

Moritz, Alexandra, und Jörn Hendrich Block. 2014. Crowdfunding und Crowdinvesting: State-ofthe-Art der wissenschaftlichen Literatur. Zeitschrift für KMU und Entrepreneurship : ZfKE 62(1): 57-89.

Nastoll, Maike. 2013. Was ist der Kommunikationsplan? https://faq.startnext.de/Projektkommunikation/ id/Was+ist+der+Kommunikationsplan+-229/modul/Projektkommunikation/aid/200. Zugegriffen am 16.08.2016.

Lachmann, Eckhard. 1988. Delphi-Methode. In WISU - Das Wirtschaftsstudium 17(6): $328 \mathrm{ff}$.

Ordanini, Andrea, Lucia Miceli, Marta Pizzetti, und A. Parasuraman. 2011. Crowdfunding: Transforming customers into investors through innovative service platforms. Journal of Service Management 22(4): 443-470.

Paivio, Allan. 1990. Mental representations. A dual coding approach, Oxford psychology series, no. 9. New York/Oxford: Oxford University Press/Clarendon Press.

Prefundia. 2013. Jeff Schwarting: Hacking Kickstarter's „Popular“ algorithm|how to create a wildly successful campaign. http://prefundia.com/blog/hacking-kickstarters-popular-algorithm-howto-become-most-popular/. Zugegriffen am 25.08.2016.

Sixt, Elfriede. 2014. Schwarmökonomie und Crowdfunding. Webbasierte Finanzierungssysteme im Rahmen realwirtschaftlicher Bedingungen. Wiesbaden: Springer Gabler.

Strickler, Yancey. 2010. The price is right. https://www.kickstarter.com/blog/the-price-is-right. Zugegriffen am 16.08.2016.

Tatikonda, M. V., und S. R. Rosenthal. 2000. Technology novelty, project complexity, and product development project execution success: a deeper look at task uncertainty in product innovation. IEEE Transactions on Engineering Management 47(1): 74-87.

Ward, Chris, und Vandana Ramachandran. 2010. Crowdfunding the next hit: Microfunding online experience goods. In Workshop on computational social science and the wisdom of crowds at NIPS2010. Lake Tahoe: Curran Associates.

Webster, Jane, und Richard T. Watson. 2002. Analyzing the past to prepare for the future: Writing a literature review. Management Information Systems Quarterly 26(2): 3.

Xu, Anbang, Xiao Yang, Huaming Rao, Wai-Tat Fu, Shih-Wen Huang, und Brian P Bailey. 2014. Show me the money! In The 32nd annual ACM conference, Hrsg. Matt Jones, Philippe Palanque, Albrecht Schmidt und Tovi Grossman, 591-600. Toronto.

Younkin, Peter, und Keyvan Kashkooli. 2013. A crowd or a community? http://funginstitute. berkeley.edu/sites/default/files/A_Crowd_Or_Community.pdf. Zugegriffen am 15.02.2016.

Zheng, Haichao, Neng Wan, Dongyu Chen, und Tao Wang. 2014. Antecedents of project implementation success in crowdfunding. In 18th PACIS, Chengdu, 1-13. Atlanta: AISEL.

Zvilichovsky, David, Yael Inbar, und Ohad Barzilay. 2013. Playing both sides of the market: Success and reciprocity on crowdfunding platforms. In Thirty fourth international conference on information systems, Milan. 